\title{
A genome-wide study of two-component signal transduction systems in eight newly sequenced mutans streptococci strains
}

\author{
Lifu Song ${ }^{1,4}$, Padhmanand Sudhakar' ${ }^{1}$, Wei Wang ${ }^{1 *}$, Georg Conrads², Anke Brock², Jibin Sun', Irene Wagner-Döbler ${ }^{3}$ \\ and An-Ping Zeng ${ }^{1 *}$
}

\begin{abstract}
Background: Mutans streptococci are a group of gram-positive bacteria including the primary cariogenic dental pathogen Streptococcus mutans and closely related species. Two component systems (TCSs) composed of a signal sensing histidine kinase (HK) and a response regulator (RR) play key roles in pathogenicity, but have not been comparatively studied for these oral bacterial pathogens.

Results: HKs and RRs of 8 newly sequenced mutans streptococci strains, including S. sobrinus DSM20742, S. ratti DSM20564 and six S. mutans strains, were identified and compared to the TCSs of S. mutans UA159 and NN2025, two previously genome sequenced S. mutans strains. Ortholog analysis revealed 18 TCS clusters (HK-RR pairs), 2 orphan HKs and 2 orphan RRs, of which 8 TCS clusters were common to all 10 strains, 6 were absent in one or more strains, and the other 4 were exclusive to individual strains. Further classification of the predicted HKs and RRs revealed interesting aspects of their putative functions. While TCS complements were comparable within the six S. mutans strains, S. sobrinus DSM20742 lacked TCSs possibly involved in acid tolerance and fructan catabolism, and S. ratti DSM20564 possessed 3 unique TCSs but lacked the quorum-sensing related TCS (ComDE). Selected computational predictions were verified by PCR experiments.
\end{abstract}

Conclusions: Differences in the TCS repertoires of mutans streptococci strains, especially those of S. sobrinus and $S$. ratti in comparison to $S$. mutans, imply differences in their response mechanisms for survival in the dynamic oral environment. This genomic level study of TCSs should help in understanding the pathogenicity of these mutans streptococci strains.

Keywords: Mutans streptococci, Streptococcus mutans, Streptococcus ratti, Streptococcus sobrinus, Two-component system, Histidine kinase, Response regulator, Signal transduction, Comparative genomics

\section{Background}

A group of oral streptococci which is closely related to the primary cariogenic dental pathogen Streptococcus mutans is referred to as "mutans streptococci", and it includes in addition to $S$. mutans also $S$. ratti, and $S$. sobrinus among others. A common feature of the mutans streptococci is their ability to metabolize carbohydrates present in our daily diet to produce energy for their survival and to produce organic acids which erode and dematerialize the tooth enamel and dentin. These oral bacteria are able to grow in a community and

* Correspondence: wei.wang@tu-harburg.de; aze@tu-harburg.de
Full list of author information is available at the end of the article colonize the oral environment by attaching to the tooth surfaces and forming biofilms. They can also tolerate and adapt to the harsh and rapidly changing physiological conditions of the oral cavity such as extreme acidity, fluctuation of nutrients, reactive oxygen species, and other environmental stresses [1]. They occasionally also cause bacteremia and infective endocarditis [2]. All together, the survival and adaptation of mutans streptococci is directly linked to their virulence and pathogenicity.

Bacterial two-component signal transduction systems (TCS) play important roles for many bacteria by enabling them to detect and respond to diverse 
changes/stresses in the environment. The conspicuous absence of TCS proteins in mammalian genomes makes them interesting potential targets for the development of novel anti-bacterial drugs. A bacterial two-component system comprises in general a transmembrane sensor histidine kinase (HK) and a corresponding cytoplasmic response regulator (RR) encoded by genes located adjacently within the same operon, although stand-alone genes coding for HKs or RRs (without a corresponding cognate $H K / R R$ in the same operon) have also been reported. In some cases, a HK and a RR are found to be merged in the same polypeptide, giving rise to a so called 'hybrid' HK protein. A HK protein is autophosphorylated at its conserved histidine (His) residue upon the recognition of a specific environmental stimulus. The phosphoryl group is then transferred to the aspartate (Asp) residue of the corresponding response regulator [3]. While HKs in general serve to detect signals, the most common function of the RRs is to bind directly to DNA and thereby modulating the expression of a certain set of genes which are necessary for mounting a physiological response to the perceived signals [3]. HK and $R R$ proteins are composed of domains which are structurally and functionally conserved and can be used for their classification.

With the advance of large scale sequencing technologies and bioinformatics tools, it has become possible to computationally predict the putative functions of genes/ proteins from the whole genome of an organism. The prediction of TCS proteins using a whole genome-based computational approach has been carried out for different organisms. Such in silico studies have broadened our understanding of genomic repertoires essential for the growth and adjustment of the organisms to altering environmental challenges [4]. The genome sequencing and annotation of the first $S$. mutans strain (UA159, serotype c) has paved the way for researchers to carry out numerous molecular biological and functional genomic studies that help in understanding the robustness, genetic specificity and complexity of this bacterium as an oral pathogen [5]. 14 TCS clusters have previously been identified in S. mutans UA159 [5,6] and many of them have been reported to be involved in its virulence, adaptation and survival [6-10]. After the sequencing of six $S$. mutans isolates, namely $S$. mutans 5 DC8, KK21, KK23, AC4446, ATCC25175 and NCTC11060, as well as S. ratti DSM20564 and S. sobrinus DSM20742, we performed a systematic identification and classification of putative TCS proteins in the genomes of these eight mutans streptococci strains, using the well annotated genome of S. mutans UA159, as well as that of $S$. mutans NN2025, as references. Furthermore, we conducted a thorough comparative analysis of the identified TCS proteins among these strains which provides valuable insights into the conservation and divergence of TCS proteins in the mutans streptococci strains studied here. PCR experiments have been carried out to verify the presence of several predicted TCS genes of particular interest.

\section{Results and discussion}

Identification of TCS proteins of the mutans streptococci strains

We have sequenced the genomes of eight mutans streptococci strains (Table 1) using the Solexa sequencing technology (genome information available at: http:// 134.28.64.65/www/index.php/genome/). The "high-quality draft" genomes obtained according to the definition by Chain et al. [11] have an overall genome coverage of $>$ 99\%, taking S. mutans NN2025 and UA159 as references. By combining the Hidden Markov Model (HMM) profiling results and the information on putative operon organization, repertoires of potential TCS proteins (HKs and RRs) in the eight newly sequenced mutans streptococci strains were obtained, as shown in Table 2 in comparison to $S$. mutans NN2025 and UA159. The total numbers of TCS proteins identified are comparable among the 10 mutans streptococci strains.

Results are presented in comparison to S. mutans UA159 and NN2025 as two reference strains. HKs and RRs are classified based on homology box analysis and output domain architecture, respectively. HPK1, HPK7, HPK8, HPK10, HPK11 represent HK families as found in the classification by Grebe et al. [15].

By analyzing the putative operon organizations of genes encoding the identified TCS proteins $98.5 \%$ of the total putative HKs and $92.2 \%$ of the total putative RR were found to constitute HK-RR pairs. Ortholog analysis of the paired or non-paired TCS proteins among the 10 mutans streptococci strains revealed a total of 18 different TCS clusters, 2 orphan HKs and 2 orphan RRs (Table 3). The numbering of the TCS clusters was based on an existing numbering system used by Levesque [12], and extended to the new TCS clusters identified in this study. Co-evolution of TCS proteins could be clearly observed. This means, HKs and RRs which belong to a particular TCS cluster are usually co-present or co-absent in a specific strain. In addition, putative alleles/orthologs of the corresponding HKs and RRs were found to be highly conserved (similarity $\geq 95 \%$ ) among the S. mutans strains. But across the species the conservation was clearly lower. Furthermore, it is obvious that, in most cases, putative HK alleles/orthologs within one TCS cluster exhibited a higher degree of diversity than the corresponding putative RR alleles/orthologs across the species. This could be attributed to the high variability of sensing/ input domains harbored by the individual HKs, as will 
Table 1 Eight newly sequenced mutans streptococci strains

\begin{tabular}{|c|c|c|}
\hline Species & Strain & Short description \\
\hline $\begin{array}{l}\text { S. } \\
\text { mutans }\end{array}$ & 5 DC8 & $\begin{array}{l}\text { Serotype c, isolated from root caries by David Beighton (London, UK), alterations in } 16 \mathrm{~S} \text { sequence in comparison to type } \\
\text { strain. }\end{array}$ \\
\hline $\begin{array}{l}\text { S. } \\
\text { mutans }\end{array}$ & AC4446 & Serotype c, isolated from a proven case of infective endocarditis in Dillingen (Germany). \\
\hline $\begin{array}{l}\text { S. } \\
\text { mutans }\end{array}$ & KK21 & Serotype c, isolated from enamel caries of an adult by Susanne Kneist (Jena, Germany), potent producer of bacteriocin. \\
\hline $\begin{array}{l}\text { S. } \\
\text { mutans }\end{array}$ & KK23 & Serotype c, isolated from enamel caries of a child by Susanne Kneist (Jena, Germany), potent producer of bacteriocin. \\
\hline $\begin{array}{l}\text { S. } \\
\text { mutans }\end{array}$ & ATCC25175 & Type strain, serotype c, isolated from carious dentine, quality control strain. \\
\hline $\begin{array}{l}\text { S. } \\
\text { mutans }\end{array}$ & NCTC11060 & Serotype $\mathrm{f}$, isolated in Denmark from a patient's blood (bacteremia), reference strain. \\
\hline S. ratti & DSM20564 & $\begin{array}{l}\text { Type strain (= ATCC19645), serotype b, isolated from caries lesion in rat, nearest neighbor to species S. mutans with a } 94-95 \% \\
\text { similarity on } 165 \text { level. }\end{array}$ \\
\hline $\begin{array}{l}\text { S. } \\
\text { sobrinus }\end{array}$ & DSM20742 & $\begin{array}{l}\text { Type strain (= ATCC33478), serotype non-d \& non-g, isolated from human dental plaque, 93\% similarity with S. mutans on } \\
16 \text { S level; considered as a relevant cariogenic species in human. }\end{array}$ \\
\hline
\end{tabular}

The species identities were determined by appliying biochemical testing (ATB 32 Strep) together with complete 16S rRNA gene sequencing.

be discussed later in the classification of HKs and RRs. A clear clustering of putative HK and RR alleles/orthologs can be visualized in the phylogenetic trees shown in Figure 1, which additionally illustrates the relationships between the different TCS clusters.
HKs are classified by two different approaches based on the homology-box analysis (classification denoted by C1) and the topology feature analysis (classification denoted by $\mathrm{C} 2$ ), respectively; RRs are classified based on the structure of their output domains (Abbreviations:

Table 2 Identification and classification of putative two component systems in the eight newly sequenced mutans streptococci strains

\begin{tabular}{|c|c|c|c|c|c|c|c|c|c|c|}
\hline Strain & $\begin{array}{l}\text { S. mutans } \\
\text { UA159 }\end{array}$ & $\begin{array}{l}\text { S. mutans } \\
\text { NN2025 }\end{array}$ & $\begin{array}{l}\text { S. mutans } \\
5 \text { DC8 }\end{array}$ & $\begin{array}{l}\text { S. } \\
\text { mutans } \\
\text { KK21 }\end{array}$ & $\begin{array}{l}\text { S. } \\
\text { mutans } \\
\text { KK23 }\end{array}$ & $\begin{array}{l}\text { S. mutans } \\
\text { AC4446 }\end{array}$ & $\begin{array}{l}\text { S. mutans } \\
\text { ATCC25175 }\end{array}$ & $\begin{array}{l}\text { S. mutans } \\
\text { NCTC11060 }\end{array}$ & $\begin{array}{l}\text { S. ratti } \\
\text { DSM20564 }\end{array}$ & $\begin{array}{l}\text { S. sobrinus } \\
\text { DSM20742 }\end{array}$ \\
\hline \multicolumn{11}{|l|}{ Identification } \\
\hline $\begin{array}{l}\text { Total TCS } \\
\text { proteins }\end{array}$ & 29 & 29 & 29 & 29 & 29 & 27 & 25 & 27 & 28 & 21 \\
\hline $\begin{array}{l}\text { Total } \\
\text { paired HKs }\end{array}$ & 14 & 14 & 14 & 14 & 14 & 13 & 12 & 13 & 13 & 9 \\
\hline $\begin{array}{l}\text { Orphan } \\
\text { HKs }\end{array}$ & 0 & 0 & 0 & 0 & 0 & 0 & 0 & 0 & 1 & 1 \\
\hline $\begin{array}{l}\text { Total } \\
\text { paired RRs }\end{array}$ & 14 & 14 & 14 & 14 & 14 & 13 & 12 & 13 & 13 & 9 \\
\hline Orphan RRs & 1 & 1 & 1 & 1 & 1 & 1 & 1 & 1 & 1 & 2 \\
\hline \multicolumn{11}{|c|}{ Classification } \\
\hline \multicolumn{11}{|l|}{ HK type } \\
\hline HPK1 & 8 & 8 & 8 & 8 & 8 & 8 & 7 & 7 & 9 & 5 \\
\hline HPK7 & 3 & 3 & 3 & 3 & 3 & 3 & 3 & 4 & 3 & 2 \\
\hline HPK8 & 1 & 1 & 1 & 1 & 1 & 1 & 1 & 1 & 1 & 1 \\
\hline HPK10 & 1 & 1 & 1 & 1 & 1 & 1 & 1 & 1 & 0 & 1 \\
\hline HPK11 & 0 & 0 & 0 & 0 & 0 & 0 & 0 & 0 & 1 & 0 \\
\hline unclassified & 1 & 1 & 1 & 1 & 1 & 0 & 0 & 0 & 0 & 1 \\
\hline \multicolumn{11}{|c|}{ RR type } \\
\hline NarL & 3 & 3 & 3 & 3 & 3 & 3 & 3 & 4 & 3 & 2 \\
\hline LytTR & 2 & 2 & 2 & 2 & 2 & 2 & 2 & 2 & 1 & 3 \\
\hline AmiR & 0 & 0 & 0 & 0 & 0 & 0 & 0 & 0 & 1 & 0 \\
\hline OmpR & 9 & 9 & 9 & 9 & 9 & 9 & 8 & 8 & 9 & 6 \\
\hline unclassified & 1 & 1 & 1 & 1 & 1 & 0 & 0 & 0 & 0 & 0 \\
\hline
\end{tabular}


Table 3 Ortholog analysis and classifications of the putative TCS proteins

\begin{tabular}{|c|c|c|c|c|c|c|c|c|c|c|c|c|c|c|}
\hline & TCSProtein & RR family & C1 & $\mathrm{C} 2$ & $\begin{array}{l}\text { S. mutans } \\
\text { UA159 }\end{array}$ & $\begin{array}{l}\text { S. mutans } \\
\text { NN2025 }\end{array}$ & $\begin{array}{l}\text { S. mutans } \\
5 \text { DC8 }\end{array}$ & $\begin{array}{l}\text { S. mutans } \\
\text { KK21 }\end{array}$ & $\begin{array}{l}\text { S. mutans } \\
\text { KK23 }\end{array}$ & $\begin{array}{l}\text { S.mutans } \\
\text { AC4446 }\end{array}$ & $\begin{array}{l}\text { S. mutans } \\
\text { ATCC } 25175\end{array}$ & $\begin{array}{l}\text { S. mutans } \\
\text { NCTC } 11060\end{array}$ & $\begin{array}{l}\text { S.ratti } \\
\text { DSM20564 }\end{array}$ & $\begin{array}{l}\text { S. sobrinus } \\
\text { DSM20742 }\end{array}$ \\
\hline \multirow[t]{2}{*}{ TCS-1 } & HK (VicK) & - & HPK1 & C & SMU.1516 & GI|290580114 & smc|01510 & smd|01560 & sme|01514 & smf|01497 & smg|01519 & smh|01557 & sra|1000270(90) & sob|6900029(74) \\
\hline & RR (VicR) & OmpR & - & & SMU.1517 & GI|290580113 & smc|01511 & smd|01561 & sme|01515 & smf|01498 & smg|01520 & smh|01558 & sra|1000269(96) & sob|6900028(87) \\
\hline \multirow[t]{2}{*}{ TCS-2 } & HK (CiaH) & - & HPK1 & E & SMU.1128 & GI|290580439 & smc|01121 & smd|01170 & sme|01262 & smf|01137 & smg|01147 & smh|01166 & sra|3300059(83) & sob|10000005(53) \\
\hline & RR (CiaR) & OmpR & - & & SMU.1129 & GI||290580438 & smc|01122 & smd|01171 & sme|01263 & smf|01138 & smg|01148 & smh|01167 & sra|3300058(94) & sob|10000006(86) \\
\hline \multirow[t]{2}{*}{ TCS-3 } & HK (CovS) & - & HPK1 & $E$ & SMU.1145c & GI|290580424 & smc|01138 & smd|01187 & sme|01279 & smf|01154 & smg|01163 & smh|01183 & sra|3300038(48) & Absent \\
\hline & RR (CovR) & OmpR & - & & SMU.1146C & GI|290580423 & smc|01139 & smd|01188 & sme|01280 & smf|01155 & smg|01164 & smh|01184 & sra|3300037(76) & Absent \\
\hline \multirow[t]{2}{*}{ TCS-4 } & HK (KinF) & - & HPK1 & $E$ & SMU.928 & GI|290580625 & smc|00919 & smd|00964 & sme|01045 & smf|00943 & smg|00948 & smh|00938 & sra|1200002(75) & sob|15200007(50) \\
\hline & RR (LlrF) & OmpR & - & & SMU.927 & GI|290580626 & smc|00918 & smd|00963 & sme|01044 & smf|00942 & smg|00947 & smh|00937 & sra|1200003(89) & sob|15200008(70) \\
\hline \multirow[t]{2}{*}{ TCS-5 } & HK (ScnK) & - & HPK1 & E & SMU.1814 & GI||290579846 & smc|01808 & smd|01870 & sme|01810 & smf|01679 & Absent & Absent & Absent & Absent \\
\hline & $\mathrm{RR}(\mathrm{ScnR})$ & OmpR & - & & SMU.1815 & GI|290579845 & smc|01809 & smd|01871 & sme|01811 & smf|01680 & Absent & Absent & Absent & Absent \\
\hline \multirow[t]{2}{*}{ TCS-6 } & HK (SpaK) & - & HPK1 & $E$ & SMU.660 & GI||290580857 & smc|00645 & smd|00684 & sme|00777 & smf|00640 & smg|00672 & smh|00668 & sra|1000132(71) & sob|3600002(47) \\
\hline & RR (SpaR) & OmpR & - & & SMU.659 & GI||290580858 & smc|00643 & smd|00682 & sme|00775 & smf|00639 & smg|00670 & smh|00666 & sra|1000130(80) & sob|3600003(67) \\
\hline \multirow[t]{2}{*}{ TCS-7 } & HK (PhoR) & - & HPK1 & $E$ & SMU.1037C & GI|290580522 & smc|01032 & smd|01081 & sme|01153 & smf|01049 & smg|01055 & smh|01052 & Absent & Absent \\
\hline & RR (YcbL) & OmpR & - & & SMU.1038c & G||290580521 & smc|01033 & smd|01082 & sme|01154 & smf|01050 & smg|01056 & smh|01053 & Absent & Absent \\
\hline \multirow[t]{2}{*}{ TCS-8 } & HK (KinG) & - & HPK1 & $M$ & SMU.1009 & GI||290580539 & smc|01005 & smd|01053 & sme|01135 & smf|01030 & smg|01036 & smh|01024 & sra|900051(80) & sob|6900007(55) \\
\hline & RR (LIrG) & OmpR & - & & SMU.1008 & GI||290580540 & smc|01004 & smd|01052 & sme|01134 & smf|01029 & smg|01035 & smh|01023 & sra|900049(89) & sob|6900006(64) \\
\hline \multirow[t]{2}{*}{ TCS-9 } & HK (LevS) & - & HPK7 & M & SMU.1965C & G||290579718 & smc|01957 & smd|02016 & sme|01953 & smf|01824 & smg|01946 & smh|01994 & sra|3500035(88) & Absent \\
\hline & RR (LevR) & NarL & - & & SMU.1964C & G||290579719 & smc|01956 & smd|02015 & sme|01952 & smf|01823 & smg|01945 & smh|01993 & sra|3500036(91) & Absent \\
\hline \multirow[t]{2}{*}{ TCS-10 } & HK (LytS) & - & HPK8 & $M$ & SMU.577 & GI|290580924 & smc|00560 & smd|00598 & sme|00685 & smf|00568 & smg|00588 & smh|00583 & sra|1000030(95) & sob|800016(80) \\
\hline & RR (LytT) & LytTR & - & & SMU.576 & GI|290580925 & smc|00559 & smd|00597 & sme|00684 & smf|00567 & smg|00587 & smh|00582 & sra|1000029(92) & sob|800017(70) \\
\hline \multirow[t]{2}{*}{ TCS-11 } & HK (LiaS) & - & HPK7 & $M$ & SMU.486 & GI|290581011 & smc|00465 & smd|00505 & sme|00595 & smf|00473 & smg|00493 & smh|00475 & sra|2900015(89) & sob|4200072(64) \\
\hline & RR (LiaR) & NarL & - & & SMU.487 & GI||290581010 & smc|00466 & smd|00506 & sme|00596 & smf|00474 & smg|00494 & smh|00476 & sra|2900013(92) & sob|4200071(82) \\
\hline \multirow[t]{2}{*}{ TCS-12 } & HK (HK11) & - & HPK7 & $M$ & SMU.1548C & GI|290580085 & smc|01542 & smd|01591 & sme|01547 & smf|01527 & smg|01550 & smh|01590 & sra|1000231(53) & sob|11300005(50) \\
\hline & RR (RR11) & NarL & - & & SMU.1547c & GI|290580086 & smc|01541 & smd|01590 & sme|01545 & smf|01526 & smg|01548 & smh|01588 & sra|1000232(82) & sob|11300004(72) \\
\hline \multirow[t]{2}{*}{ TCS-13 } & HK(ComD) & - & HPK10 & $M$ & SMU.1916 & G||290579761 & smc|01910 & smd|01968 & sme|01906 & smf|01777 & smg|01900 & smh|01947 & Absent & sob|8500005(37) \\
\hline & RR (ComE) & LytTR & - & & SMU.1917 & GI||290579760 & smc|01911 & smd|01969 & sme|01907 & smf|01778 & smg|01901 & smh|01948 & Absent & sob|8500004(43) \\
\hline \multirow[t]{2}{*}{ TCS-14 } & $\mathrm{HK}$ & - & $?$ & C & SMU.45 & GI|290579565 & smc|00046 & smd|00053 & sme|00049 & Absent & Absent & Absent & Absent & Absent \\
\hline & $\mathrm{RR}$ & $?$ & - & & SMU.46 & GI||290579566 & smc|00047 & smd|00054 & sme|00050 & Absent & Absent & Absent & Absent & Absent \\
\hline \multirow[t]{2}{*}{ TCS-15 } & HK(ComP) & - & HPK7 & $M$ & Absent & Absent & Absent & Absent & Absent & Absent & Absent & smh|00177 & Absent & Absent \\
\hline & $\mathrm{RR}(\mathrm{CmpR})$ & NarL & - & & Absent & Absent & Absent & Absent & Absent & Absent & Absent & $\operatorname{smh} \mid 00178$ & Absent & Absent \\
\hline \multirow[t]{2}{*}{ TCS-16 } & $\mathrm{HK}$ & & HPK1 & E & Absent & Absent & Absent & Absent & Absent & Absent & Absent & Absent & sra|800020 & Absent \\
\hline & $\mathrm{RR}$ & OmpR & & & Absent & Absent & Absent & Absent & Absent & Absent & Absent & Absent & sra|800019 & Absent \\
\hline \multirow[t]{2}{*}{ TCS-17 } & $\mathrm{HK}$ & & HPK1 & $E$ & Absent & Absent & Absent & Absent & Absent & Absent & Absent & Absent & sra|3500015 & Absent \\
\hline & RR & OmpR & & & Absent & Absent & Absent & Absent & Absent & Absent & Absent & Absent & sra|3500014 & Absent \\
\hline
\end{tabular}


Table 3 Ortholog analysis and classifications of the putative TCS proteins (Continued)

\begin{tabular}{|c|c|c|c|c|c|c|c|c|c|c|c|c|c|c|}
\hline \multirow[t]{2}{*}{ TCS-18 } & \multicolumn{2}{|l|}{ HK } & \multirow[t]{2}{*}{ HPK11 } & \multirow[t]{2}{*}{ C } & \multirow{2}{*}{$\begin{array}{l}\text { Absent } \\
\text { Absent }\end{array}$} & \multirow{2}{*}{$\begin{array}{l}\text { Absent } \\
\text { Absent }\end{array}$} & \multirow{2}{*}{$\begin{array}{l}\text { Absent } \\
\text { Absent }\end{array}$} & \multirow{2}{*}{$\begin{array}{l}\text { Absent } \\
\text { Absent }\end{array}$} & \multirow{2}{*}{$\begin{array}{l}\text { Absent } \\
\text { Absent }\end{array}$} & \multirow{2}{*}{$\begin{array}{l}\text { Absent } \\
\text { Absent }\end{array}$} & \multirow{2}{*}{$\begin{array}{l}\text { Absent } \\
\text { Absent }\end{array}$} & \multirow{2}{*}{$\begin{array}{l}\text { Absent } \\
\text { Absent }\end{array}$} & \multirow{2}{*}{$\begin{array}{l}\text { sra|1400052 } \\
\text { sra|1400053 }\end{array}$} & \multirow{2}{*}{$\begin{array}{l}\text { Absent } \\
\text { Absent }\end{array}$} \\
\hline & $\mathrm{RR}$ & AmiR & & & & & & & & & & & & \\
\hline Orphan HK1 & HK & & HPK1 & $\mathrm{N}$ & Absent & Absent & Absent & Absent & Absent & Absent & Absent & Absent & sra|3800008 & Absent \\
\hline Orphan HK2 & HK & & $?$ & M & Absent & Absent & Absent & Absent & Absent & Absent & Absent & Absent & Absent & sob|13200014 \\
\hline Orphan RR1 & RR (GcrR) & OmpR & - & & SMU.1924 & G||290579753 & smc|01920 & smd|01978 & sme|01916 & smf|01787 & smg|01910 & smh|01957 & sra|200007(87) & sob|100018(55) \\
\hline Orphan RR2 & & LytTR & & & Absent & Absent & Absent & Absent & Absent & Absent & Absent & Absent & Absent & sob|14100005 \\
\hline
\end{tabular}



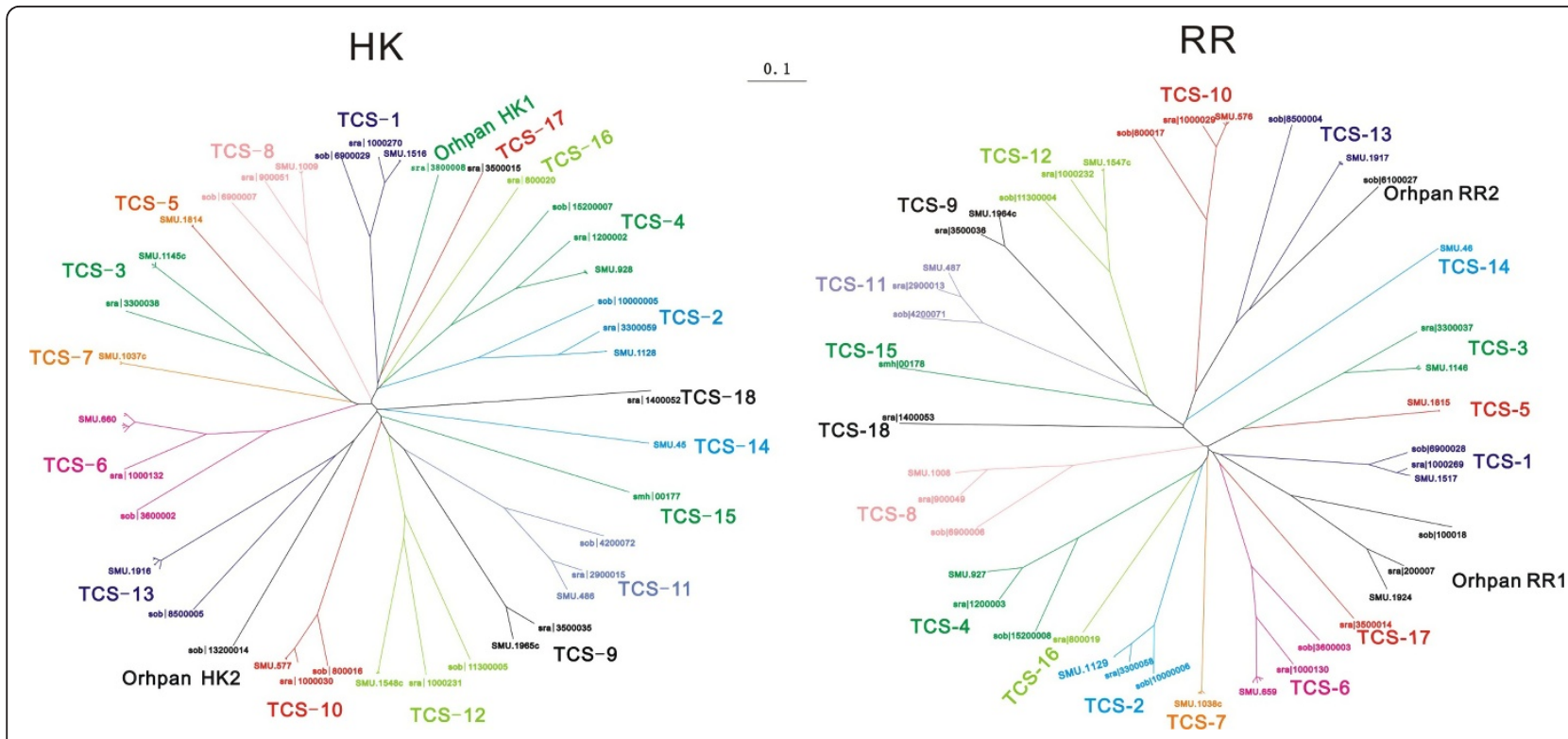

Figure 1 Phylogenetic trees of the identified putative HKs and RRs in the eight newly sequenced mutans streptococci strains and the two reference strains S. mutans UA159 and NN2025. The trees were constructed using ClustalX by applying the neighbor-joining method. The scale bar is shown above the trees and the scale is in units of "substitution/site".

$H P K$, histidine protein kinase; $C, E$ and $M$ stand for cytoplasmic, extracytoplasmic and membrane sensing, respectively; $N$ denotes that no sensing mechanism could be predicted either due to the complete absence or a truncation of the predicted sensory domain; $S M U$ represents the proteins corresponding to the ORFs of $S$. mutans UA159, GI of $S$. mutans NN2025; smc of $S$. mutans 5 DC8; smd of $S$. mutans KK21; sme of $S$. mutans KK23; smf of $S$. mutans AC4446; smg of $S$. mutans ATCC 25175 ; smh of S. mutans NCTC 11060; sra of S. ratti DSM 20564; sob of S. sobrinus DSM 20742. The italic numbers in parentheses stand for similarities in percentage of the TCS proteins in S. ratti DSM20564 and S. sobrinus DSM20742 compared to the corresponding orthologs in S. mutans UA159. All other abbreviations are referred to in Table 2).

Eight of the 18 TCS clusters (designated as TCS-1, TCS-2, TCS-4, TCS-6, TCS-8, TCS-10, TCS-11, TCS12) were common to all the strains. Six clusters (TCS-3, TCS-5, TCS-7, TCS-9, TCS-13, TCS-14) were observed to be absent in one or several strains. An interesting observation was that $S$. ratti DSM20564 lacked any homologs of the sensor histidine kinase and the cognate response regulator of TCS-13 (known as ComD and ComE for S. mutans UA159). On the other hand, TCS16, TCS-17 and TCS-18 were found to be unique to $S$. ratti DSM20564. TCS-15, a putative ortholog of a quorum sensing-related TCS cluster well studied in the gram-positive bacterium Bacillus subtilis, was uniquely identified in S. mutans NCTC11060, a serotype f blood isolate.
Two orphan HKs were identified as uniquely present in S. ratti DSM20564 (sra|3800008, named as orphan HK1) and S. sobrinus DSM20742 (sob|13200014, orphan HK2), respectively. In contrast, an orphan RR (orphan RR1, SMU.1924 in S. mutans UA159) was found to be common to all the 10 strains. S. sobrinus DSM20742 harbored an additional unique orphan RR (sob| 14100005, orphan RR2).

No hybrid HKs could be detected in all the genomes of the 10 mutans streptococci strains compared in this study. Also, the more complex variant of the two-component system, known as the phosphor-relay system comprised of an autophosphorylatable hybrid HK, one or more histidine phosphotransferases (HPT) and a terminal RR was totally absent, because no HPT domains were found in these strains.

\section{Classification of the histidine kinases}

HKs are multi-domain homodimeric proteins consisting of a more variable $\mathrm{N}$-terminal for recognition of diverse signals from different cellular localizations (extracellular, transmembrane or intracellular) and a more conserved $\mathrm{C}$-terminal containing the conserved histidine residue for signal transduction to its corresponding RR protein. The N-terminal regions of most HKs are characterized by the presence of one to several (up to 20) transmembrane helices (although exceptions to this rule could also be found [13]), whereas the $\mathrm{C}$-terminal region harbors characteristic domain profiles such as HATPase_c and His_kinase domains [3,14]. In addition, HK proteins exhibit characteristic sequence motifs (homology boxes), 
including the $\mathrm{H}-, \mathrm{N}-, \mathrm{D}-, \mathrm{F}-$ and $\mathrm{G}$ - boxes, as a result of the conservation of the histidine $(\mathrm{H})$, aspargine $(\mathrm{N})$, aspartate $(D)$, phenylalanine $(F)$ and glycine $(G)$ residues at fixed positions. In addition to the H-box, which contains the autophosphorylatable histidine residue functionally essential for a histidine kinase, other homology boxes are also presumed to play crucial roles in different biological processes. For example, the $\mathrm{D}$-box is suggested to be responsible for DNA binding and the Gbox is believed to be involved in phosphotransfer reactions. Homology boxes have been used to define various HK families [15]. Thus, to better understand the functions of individual HK proteins of the mutans streptococci strains, two classification approaches were applied in this study to the putative HK proteins. The first approach termed here as homology box analysis was based on the conserved sequence motifs described before by Grebe et al. [15]. This classification scheme sorts HK proteins into 11 different families (HPK1 to HPK11) depending on the sequence motifs of the various homology boxes identified by multi-alignment. Thus, members of the different families can be distinguished by conserved patterns in each of the homology boxes. The second approach termed here as domain architecture analysis was based on the classification scheme proposed by Mascher et al. [13], which considers the conservation of domain architecture of an entire HK, such as transmembrane helices, linker regions, sensing domains and transmitter domains. This classification method is especially helpful in understanding the signal sensing mechanisms of HKs.

\section{Classification of HKs based on homology box analysis}

Five HK families (HPK1, HPK7, HPK8, HPK10, HPK11) were recognized among the 10 mutans streptococci strains. For each of the 10 strains the distributions of their HKs within these families are highlighted in Table 2 and visualized in Figure 2 by multiplesequence alignments of the HK proteins. It is clear that the amino acid residues surrounding the conserved histidine residue in the $\mathrm{H}$-box are more conserved compared to those flanking the aspargine, aspartate, phenylalanine and glycine residues in the $\mathrm{N}$-, D-, F-, and G-boxes, respectively.

The assignment of HKs of all the 18 TCS clusters to the homology box-based HK families is given in Table 3. HKs of TCS- 1 to TCS-8, TCS-16 and TCS-17 and the orphan HK of S. ratti DSM20564 (sra|3800008, orphan HK1) were grouped into the HPK1 family, accounting for more than $50 \%$ of the total HKs identified. HPK1 family has been known as the most common type among the histidine kinase families, characterized by highly conserved aspartate/glutamate, lysine/arginine and proline residues downstream of the conserved histidine residue in the $\mathrm{H}$-boxes (Figure 2). The orphan HK1 might be a 'dead' gene because of the lack of a sensing domain, which can be inferred from the relatively short $\mathrm{N}$-terminal region prior to the $\mathrm{H}$-box, as highlighted in Figure 2. Moreover, 4 residues including the most important histidine residue in the H-box were also found to be missing in orphan HK1.

The second most abundant HK family was the HPK7 family, which contained more than $20 \%$ of all the HKs identified and included HKs of 4 different TCS clusters, namely TCS-9, TCS-11, TCS-12 and TCS-15. As shown in Figure 2, the most prominent feature of this HK family was the conservation of negatively charged aspartate residue downstream of the histidine residue and the positively charged arginine residue upstream of the histidine residue in the H-box.

Two other HK families found were the HPK8 and the HPK10 families. The HPK8 family comprised only HKs of the TCS-10 cluster. Known as LytS in $S$. mutans UA159, HKs of this family were highly conserved across all the 10 strains, as evidenced by the highly conserved sequence patterns of the $\mathrm{H}$ - and $\mathrm{N}$ boxes (Figure 2). The identification of HKs belonging to the HPK8 family was made possible by aligning the corresponding HKs of the mutans streptococci strains of this study with their BLASTp homologs. This enabled the unambiguous verification of the conservation of homology boxes characteristic of the HPK8 family. The HPK10 family has been reported to include kinases that regulate competence for genetic transformation in Streptococcus spp. [15]. Indeed, HKs of TCS-13 (known as ComD for S. mutans UA159) fell within this family. This family was characterized by the presence of a tyrosine located two residues downstream of the conserved histidine residue, the absence of the H-box proline and the lack of the D and Fboxes. HK of TCS-13 (ComD) was absent in S. ratti DSM20564, which lacked any orthologs of both ComD and its cognate response regulator ComE.

The last HK family identified is the HPK11 family containing only one histidine kinase uniquely found in S. ratti DSM20564. A similar strategy as that used for the classification of HKs of the HPK8 family was performed by using BLASTp homologs to recognize conserved homology boxes. The H-box of this family was quite distinct from the H-boxes of other families in that the conserved histidine was flanked at its left by another histidine residue, in addition to the downstream arginine $(\mathrm{R})$ and asparagine $(\mathrm{N})$ residues characteristic of this family (Figure 2).

The HK of TCS-14 and the unique orphan HK of the strain S. sobrinus DSM20742 (sob|13200014, orphan HK2) could not be classified into any of the $11 \mathrm{HK}$ families. 


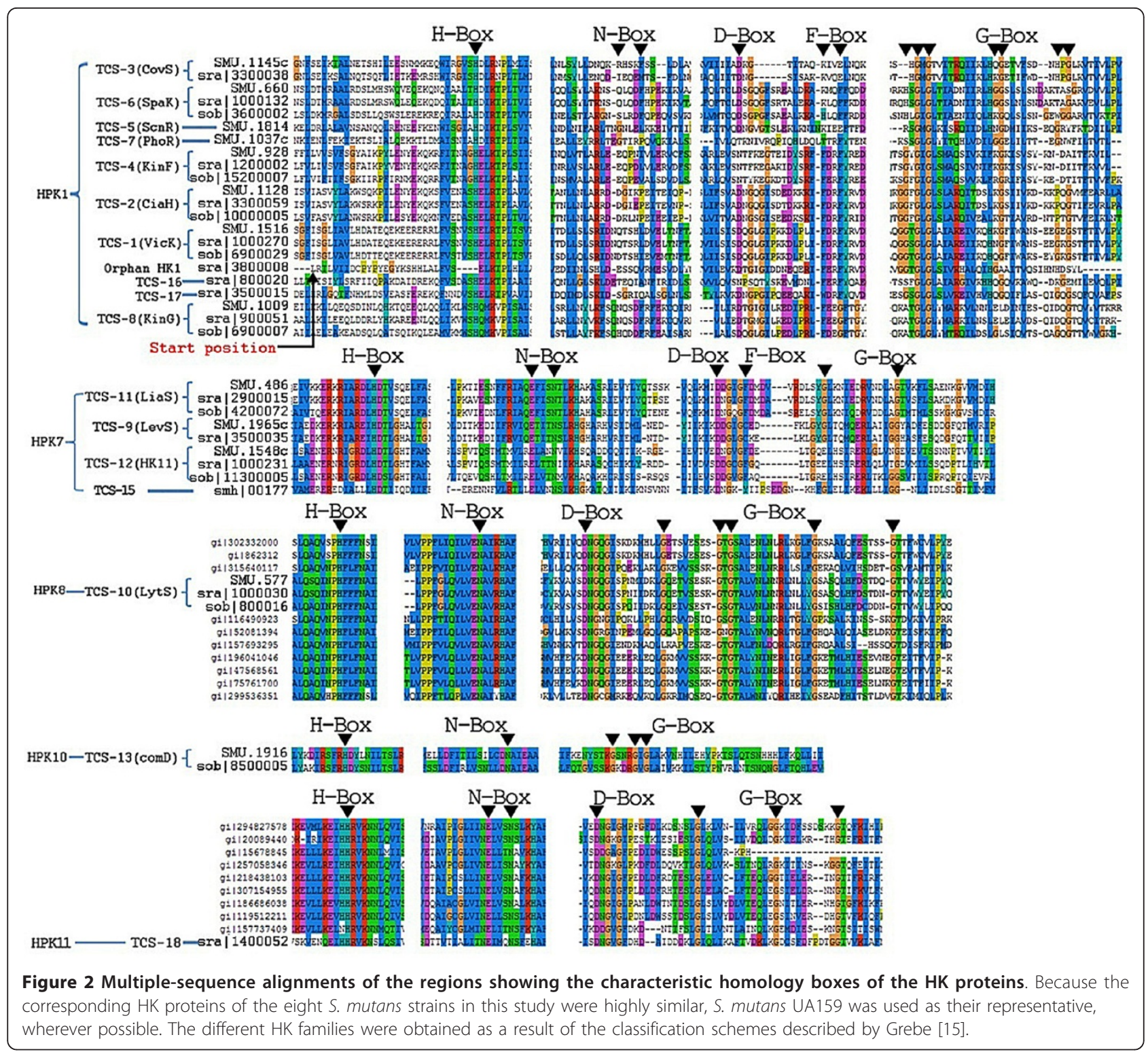

\section{Classification of HKs based on domain architecture analysis}

The homology boxes-based classification of HKs described above focused solely on the conserved sequence motifs in the C-terminal transmitter domains of histidine kinases. Since the biological function of TCS-mediated signal transduction manifests itself in signal input and output rather than in the communication between its two components, it is necessary to characterize the $\mathrm{N}$-terminal sensory regions with respect to the signal sensing mechanism of a particular HK protein. To this end, the classification method proposed by Mascher et al. [13] was applied to group the putative HKs into three different groups: extracytoplasmic-sensing HKs, cytoplasmic-sensing HKs, and membrane- sensing HKs (HKs with sensing mechanisms associated with membrane-spanning helices), as shown in Figure 3.

Extracytoplasmic sensing HKs represent the largest group of membrane-bound sensor kinases. Among all the HKs identified, HKs of TCS-2 to TCS- 6 as well as TCS-16 and TCS-17 were recognized as extracytoplasmic sensing HKs by displaying at the $\mathrm{N}$-terminal region an extracytoplasmic putative signal perception domain composed of 110-140 amino acids, which were flanked by (at least) two transmembrane helices (TMs). The cytoplasmic part of the HK proteins harboring the transmitter domain comprised either a HisKA-HATPase_c domain (HKs of TCS-2, TCS-3, TCS-4, TCS-5 and TCS-16) or a HAMP-HisKA-HATPase_c domain (HKs of TCS-6 and TCS-17). HisKA and HATPase_c are 


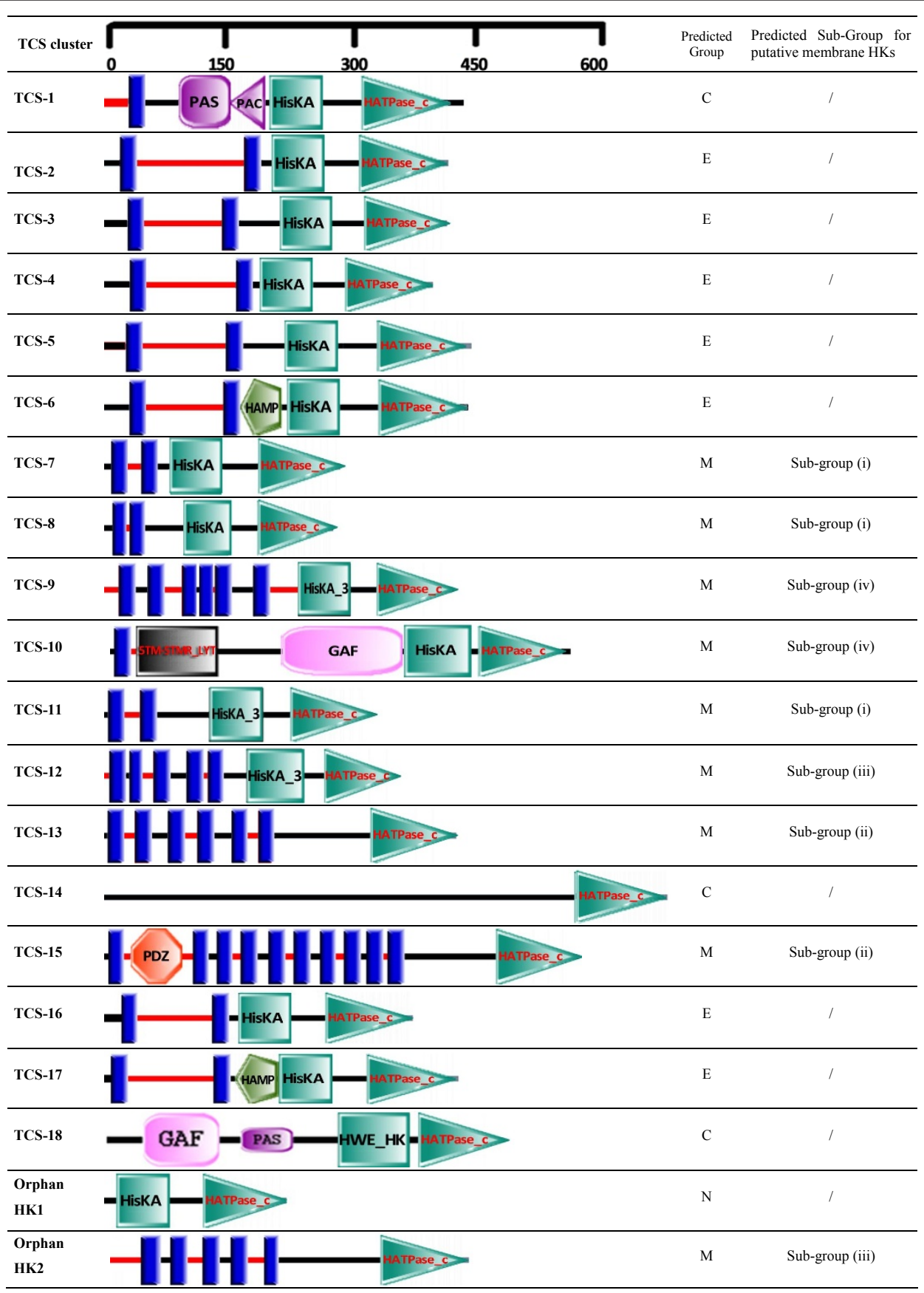

Figure 3 Domain architectures of histidine kinases representative of each TCS clusters. The pictorial depiction is based on the predictions carried out using the SMART web interface http://smart.embl-heidelberg.de/ with small manual modifications. The transmembrane helices (TMs), represented by blue bars, and the cellular localization of the linker regions between the helices were predicted using the tool TMHMM [89]. Red and black horizontal lines depict extracytoplasmic and cytoplasmic regions, respectively. The locations of the conserved domains within the protein sequences are represented by the labeled symbols. Domain definitions are according to Pfam http://pfam.sanger.ac.uk: HAMP, linker domain presenting in histidine kinase, adenylyl cyclases, methyl binding proteins and phosphatases (PF00672); HisKA, dimerisation/ phosphoacceptor domain of histidine kinase (PF00512); HATPase_c, HK-type ATPase catalytic domain (PF02518); HisKA_2, dimerisation/ phosphoacceptor domain of a sub-family of histidine kinases (PF07568); HisKA_3, dimerisation/phosphoacceptor domain of a sub-family of histidine kinases (PF07730); PAS, signal sensor domain (PF00989); PAC (PF00785), a motif often found at the C-terminal side of the PAS domain and proposed to contribute to the PAS domain fold, GAF, cytoplasmic signaling domain (PF01590); PDZ, also known as DHR or GLGF (PF00595), a signal sensor domain of diverse functional specificities. All other abbreviations are referred to in Table 2 and Table 3. $\underline{C}, \underline{E}$ and $\underline{M}$ stand for cytoplasmic, extracytoplasmic and membrane sensing, respectively; $\mathrm{N}$ denotes no sensing mechanism could be predicted due to either the complete absence or a truncation of the predicted sensory domain. 
domains essential for the functionality of a histidine kinase. HAMP functions as a linker to bridge the transmembrane helix and the transmitter domain [16]. It should be noticed that sequence regions containing about 110 residues were found to exist between the TM region and the HisKA domain of the TCS- 3 and TCS- 5 HKs. We suspect that these HKs could possibly contain unknown linker domains within this region which cannot be identified due to the currently limited knowledge on HK linker domains.

Cytoplasmic-sensing HKs include either membraneanchored or soluble proteins with their sensing domains located inside the cytoplasm [13]. Only HKs of three TCS clusters, namely the membrane anchored HK of TCS-1 and the soluble HKs of TCS-14 and TCS-18, were identified as HKs with putative cytoplasmic sensing functions. A more detailed look at the HKs of TCS-14 and TCS-18 showed that they were predicted to be completely intracellular proteins. The HK of TCS-1, known as VicK in $S$. mutans UA159, is typified by the presence of only one membrane-anchoring transmembrane helix and a very short extracytoplasmic linker containing 30 amino acids in the N-terminal region. In addition, both HKs of TCS-1 and TCS-18 possess a PAS domain. PAS domains are known to play important roles as sensory modules for sensing oxygen tension, cellular redox state, or light intensity [17]. PAS domain-containing proteins have been reported to be involved in oxidative stress response [18], competence [19], virulence [20] and biofilm formation [21] in many organisms. Most PAS domain-containing proteins are intracellularly located with dual functions of monitoring both the external and internal environments by perceiving alterations in the electron transport system caused by intracellular or extracellular changes in redox potential [22]. It has been reported that PAS domains are often associated with PAC domains and they are directly linked and together form the conserved 3D PAS fold [23], as also exemplified by the HK of TCS- 1 in this study. In fact, in agreement with the function of the PAS-PAC domains, the HK of TCS-1, VicK, has been reported to play a role in oxidative stress response [24]. On the other hand, the HK of TCS-18 did not contain a PAC motif but a GAF domain, which has been found to be present in combination with multiple domains in many phytochromes and cGMP-specific phosphoesterases [25]. A large variety of bacterial proteins such as those involved in signal transduction, cell autolysis, chemotaxis, glucose metabolism, nitrogen fixation, and gene regulation, as well as phototransducing proteins in plant and archea were found to contain a GAF domain [26].

The highly diverse group of HKs with sensing mechanisms linked to transmembrane regions (membrane-sensing HKs) is marked by the presence of 2 to 20 transmembrane helices implicated in signal perception. Such HKs lack a recognizable extracellular input domain. Signal sensing is either membrane associated or occurs directly within the membrane interface [13]. Although it has been suggested that this group represents the smallest group of membrane-bound sensor histidine kinases when compared to other HK families in the SMART database [13], HKs of TCS-7 to TCS-13 and TCS-15 in this study were all found to belong to this group, indicating that a relatively high percentage of HKs of the mutans streptococci strains are involved in sensing signals directly associated with the membrane. Such signals can be changes in the mechanical properties of the cell envelope, signals derived from membrane-bound enzymes or other membrane-integral components, ions, electrochemical gradients, transport processes, or the presence of compounds that affect cell envelope integrity [13]. Most quorum sensing HKs from gram-positive bacteria fall into this category, like ComD, the HK of TCS-13. Putative alleles/orthologs of ComD were found in most of the mutans streptococci strains of this study, characterized by 5 to 7 transmembrane helices in the $\mathrm{N}$-terminal region succeeded by a transmitter region containing only the HATPase_c domain. In $S$. sobrinus DSM20742 the unique orphan HK2 (sob| 13200014) was also assigned to this group. Orphan HK2 shared a sequence similarity of about $37 \%$ with the HK (sob|8500005) of TCS-13, which is the predicted ortholog of ComD in S. sobrinus DSM20742.

For the highly diverse membrane-sensing HK family, six subfamilies have been additionally defined based on features such as the number of TM helices, the type and sequential arrangement of sensory domains, the length of the inter-helix linker regions and the presence or absence of extracytoplasmic linker regions [13]. By applying this grouping scheme, the membrane-sensing HKs of TCS-7, TCS- 8 and TCS- 11 were grouped into subgroup (i). This subgroup is comprised of small sensor kinases containing 2 TM helices and lacking significant extracytoplasmic regions. HKs of this subgroup are involved in sensing cell envelope stress or mediating $\mathrm{ABC}$ transporter-coupled detoxification processes in gram-positive bacteria [27,28]. HKs of TCS-13 and TCS-15 were assigned to subgroup (ii), which is primarily comprised of quorum-sensing HKs of gram-positive bacteria possessing 6 to 10 TM helices. HKs of TCS-12 and the orphan HK2 were grouped into subgroup (iii) represented by DesK-like thermo sensors with 4 or 5 TM helices that sense membrane fluidity. HKs of TCS-9 and TCS-10 were assigned to subgroup (iv) with a conserved but yet functionally unknown input domains containing 6 to 8 TM helices.

\section{Classification of the response regulators}

A RR protein, most commonly a transcription factor [3], comprises an $\mathrm{N}$-terminal regulatory domain (receiver 
domain) and a C-terminal effector domain (output domain). The phosphorylated aspartate residue falls within the receiver domain whose structure is well conserved as represented by the Response_reg domain among almost all the known RRs [29]. Phosphorylation of the aspartate residue activates the DNA binding properties of the output domain, ultimately resulting in the direct or indirect activation or repression of a set of genes. The output domains are less conserved and differ according to the nature of the signal outputs. Hence, a classification of RRs based on the domain architecture of the C-terminal effector domain should provide insight into the specific biological function of the TCSs [30]. Despite the great variety of output domains and domain combinations, about $60 \%$ of all known response regulators belong to the OmpR, NarL or NtrC families, and all of which contain a helix-turn-helix (HTH) DNA-binding output domain. RRs with the DNA-binding output domains LytTR, AraC, Spo0A, Fis, YcbB, RpoE, and MerR account for an additional 6\% [30]. The rest of the RRs are characterized by the presence of a stand-alone REC domain (14\%) or combinations (20\%) comprising a variety of enzymatic domains, RNA-binding domains, protein or ligand-binding domains, or other newly described domains of unknown functions. The diversity of the domain architectures and the abundance of domain combinations of the output domain allow bacterial cells to regulate a variety of biological processes such as transcription, enzyme activity and protein-protein interaction in response to a number of environmental signals and stimuli [30].

The majority of the putative RRs identified in this study were classified into the following 4 families: NarL, LytTR, AmiR and OmpR, with RRs of the OmpR family constituting the largest group. For each of the $10 \mathrm{com}$ pared strains the distributions of their RRs within these families are highlighted in Table 2. The assignment of RRs of the 18 TCS clusters and the orphan RRs to the corresponding RR families is given in Table 3 .

While most RRs contain a DNA binding domain, there were two exceptions: the putative RR of TCS-14 lacked any currently known output domains and therefore could not be assigned to any of the known RR families. The RR (sra|1400053) of TCS-18, a unique TCS cluster of $S$. ratti DSM20564, contained a RNAbinding output domain and was found to be an AmiRlike response regulator. The AmiR family is a relatively newly defined response regulator family possessing RNA-binding activity. In contrast to the commonly known phosphorylation mechanism in DNA-binding RRs, AmiR is normally controlled by ligand-regulated activation, in keeping with the substitution of the conserved aspartate phosphoryl acceptor residue by serine, as well as the replacements of additional amino acid residues required for phosphoryl transfer [31]. However, unlike the classical AmiR-like proteins, residues involved in phosphoryl transfer are conserved in the RR of TCS18. In addition, we have identified its cognate HK (sra| 1400052). Therefore, we believe that RR of TCS-18 is most likely activated by its cognate HK through phosphorylation.

RRs of the OmpR family constituted the largest group (Table 2). Proteins of the OmpR family have been reported to mediate a wide range of biological functions related to, for example, osmolarity, phosphate assimilation, antibiotic resistance, virulence and toxicity [32]. NarL proteins differ from those of the OmpR family due to the presence of the LuxR_C-like DNA-binding HTH domain. Members of the NarL family have been documented to control expression of genes related to nitrogen fixation, sugar phosphate transport, nitrate and nitrite metabolism, quorum sensing, and osmotic stress etc. [33]. Proteins of the LytTR family are characterized by having a non-HTH DNA binding domain [34]. They account for about $3 \%$ of all the prokaryotic RRs [30]. Despite being found in prokaryotic genomes in small numbers when compared to other RR families, the LytTR type RR proteins have been reported to modulate the expression of many genes coding for virulence factors, fimbriae, cell wall components, bacteriocins, extracellular polysaccharides etc. [35]. Also of interest is the fact that RRs belonging to $\mathrm{NtrC}, \mathrm{AraC}, \mathrm{SpoOA}$, Fis, YcbB, RpoE and MerR families, which often appear in other bacterial genomes, are totally absent in the mutans streptococci strains compared in this study.

\section{Co-occurrence of certain HK and RR families}

The homology box-based classification of HK proteins has been shown to be able to reveal the co-occurrence relationships between the various kinase families and regulator families [15]. In other words, specific families of HK were preferentially associated with specific families of RR proteins. In this study, certain pairing combinations between the HK families and the RR families were also observed in the mutans streptococci strains as shown in Table 3. For instance, all HKs of the HPK1 family are paired with corresponding RRs of the OmpR family, and all HKs of the HPK7 family constitute TCS clusters with RRs of the NarL family. HKs of both the HPK8 and HPK10 kinase families prefer RRs of the LytTR family as their corresponding cognate response regulators. We reckon that such combinations may play a significant role in the phenomenon of crosstalking between unpaired HKs and RRs, e.g. a HK of the HPK1 family is highly likely to cross-phosphorylate an unpaired $R R$ of the OmpR family. Cross-talking mechanisms have been reported to exist in many bacterial species [36] and have also been speculated to appear 
in Streptococcus mutans strains [37]. Such a strategy could be advantageous for mutans streptococci strains to maximally utilize their relatively small complement of TCSs for processing diverse signals so as to ensure a flexibly coordinated response to the constantly changing environments. For example, while a viable knock-out mutant of the vicK gene coding for the HK of TCS-1 could be obtained, the cognate response regulator VicR has been found to be essential for the survival of $S$. mutans UA159 [38]. It has also been reported that no viable vicR knockout mutants could be obtained for Streptococcus pneumoniae, but knockout of the corresponding histidine kinase gene vicK is possible, because vicR can be phosphorylated in vivo by vanS, a gene coding for a TCS histidine kinase from Enterococcus faecium, which helps to suppress some effects of a vicK kinase disruption in S. pneumoniae [39]. According to homology box analysis the histidine kinase VanS of Enterococcus faecium belongs to HPK1 family like VicK. Thus, we speculate that such cross-phosphorylation of VicR by a non-cognate HK most likely also holds true in S. mutans UA159, and probably also for other mutans streptococci strains. Clearly, the systematic classification of HKs and RRs carried out in this study reveals potential cross-talking candidates that will be helpful in the future studies of two-component systems.

\section{Putative biological functions of the identified TCS proteins}

In the following sections, possible biological functions and significance of the TCS genes/proteins, which were common or specific to the 10 mutans streptococci strains as presented in Table 3, are discussed. To this end and for the purpose of a better overview, we have briefly summarized available knowledge on the biological functions of the TCS genes/proteins of the best studied S. mutans strain UA159. In case no knowledge is available about the functions of some TCSs, information about putative orthologs of the corresponding TCS genes/proteins in organisms other than $S$. mutans UA159 are provided (Table 4).

\section{TCS proteins common to all the 10 mutans streptococci strains}

Proteins of the TCS clusters 1, 2, 4, 6, 8, 10, 11, 12 and the orphan RR1 are common to all the 10 mutans streptococci strains compared here, indicating probably the functional importance of these TCS clusters for the adaptation and survival of these mutans streptococci (Table 4). For instance, Orphan RR1 is highly conserved across the 10 mutans streptococci strains. In S. mutans UA159, this orphan RR is encoded by gcrR (SMU.1924c) and has been found to play a vital role in sucrosedependent adherence and cariogenesis [66]. Therefore, it is conceivable that conservation of this gene across the mutans streptococci strains is essential for their primary pathogenicity.

\section{TCS proteins uniquely present/absent in one or several strains}

The TCS-3 (CovSR) cluster was predicted to be absent in S. sobrinus DSM20742. CovSR is involved in the acid tolerance response of $S$. mutans UA 159 [12], and has also been reported to be involved in counteracting oxidative stress and reducing susceptibility to phagocytic killing [46]. TCS-9 (LevRS), which affects the acid tolerance response as well [12], was also absent in S. sobrinus DSM20742. The absence of the covS and levS genes was experimentally supported by the PCR results shown in Figure 4. In S. mutans UA159, the levRS gene cluster is flanked by levQ and levT, which code for two carbohydrate-binding proteins. These four genes together constitute a four-component signal transduction system levQRST controlling the transcription of the fructan hydrolase gene (fruA) and a four-gene cluster levDEFG, which encode a fructose/mannose sugar:phosphotransferase system located immediately downstream of levQRST [55]. S. sobrinus was also found to lack the levQ, levT and levDEFG genes. Taking together, these findings indicate dramatic differences in the regulation of fructan catabolism and the acid tolerance response of S. sobrinus DSM20742 in comparison to the S. mutans strains.

It should be pointed out that the TCS nomenclature is unfortunately inconsistent among the published articles on TCSs of S. mutans strains. Many publications [67-72] on CovR actually address the orphan response regulator RR1 which is known as GcrR (SMU.1924) in S. mutans UA159 [66]. On the other hand, CovSR of $S$. mutans has also been confusingly named as ScnKR $[12,46]$. In our study, the nomenclature of TCS genes/ proteins was based primarily on the Oralgen Pathogen Sequence Database http://www.oralgen.lanl.gov. In cases where several names were given for a gene in Oralgen, gene names used by Biswas et al. [6] were preferably used.

TCS-5 (ScnKR) could be neither found in the two $S$. mutans strains ATCC25175 and NCTC11060 nor in S. ratti DSM20564 and S. sobrinus DSM20742. The asymmetric distribution of TCS-5 was also observed in a previous study, in which TCS-5 was found to be present only in two of the $10 \mathrm{~S}$. mutans strains compared [6]. In S. mutans UA159, an insertion mutant of scnK gene displayed no significant difference to the wild type with respect to growth under various stress conditions [6]. In Streptococcus pyogenes, ScnKR was found to be essential for the production of a bacteriocin (SAFF22) [49]. By a closer look at the genes in the neighborhood of ScnKR 
Table 4 A brief summary of known/putative functions of the TCSs identified in S. mutans UA159

\begin{tabular}{|c|c|c|c|c|}
\hline $\begin{array}{l}\text { TCS } \\
\text { Cluster }\end{array}$ & $\begin{array}{l}\text { TCS } \\
\text { protein }\end{array}$ & $\begin{array}{l}\text { GenBank } \\
\text { Locus } \\
\text { Tag }\end{array}$ & Functions & References \\
\hline TCS-1 & $\begin{array}{l}\text { HK-Vick } \\
\text { RR-VicR }\end{array}$ & $\begin{array}{l}\text { SMU.1516 } \\
\text { SMU.1517 }\end{array}$ & $\begin{array}{l}\text { Biofilm development, competence development, oxidative stress tolerance, acid tolerance, } \\
\text { autolysin production, glucan metabolism, fructan metabolism. }\end{array}$ & {$[7,8,10,24,40,42,43]$} \\
\hline TCS-2 & $\begin{array}{l}\text { HK-CiaH } \\
\text { RR-CiaR }\end{array}$ & $\begin{array}{l}\text { SMU.1128 } \\
\text { SMU.1129 }\end{array}$ & $\begin{array}{l}\text { Sucrose-dependent biofilm formation, competence development, multiple stress response, } \\
\text { bacteriocin production }\end{array}$ & {$[12,44,45]$} \\
\hline TCS-3 & $\begin{array}{l}\text { HK-CovS } \\
\text { RR-CovR }\end{array}$ & $\begin{array}{l}\text { SMU.1145 } \\
\text { SMU.1146 }\end{array}$ & Acid tolerance, hydrogen peroxide resistance, murine macrophage killing. & {$[12,46]$} \\
\hline TCS-4 & $\begin{array}{l}\text { HK-KinF } \\
\text { RR-LlrF }\end{array}$ & $\begin{array}{l}\text { SMU.928 } \\
\text { SMU.927 }\end{array}$ & Acid tolerance, $\mathrm{pp}(\mathrm{G}) \mathrm{pp}$ metabolism, control of alarmone synthesis. & {$[47,48]$} \\
\hline TCS-5 & $\begin{array}{l}\text { HK- } \\
\text { ScnK } \\
\text { RR- } \\
\text { ScnR }\end{array}$ & $\begin{array}{l}\text { SMU.1814 } \\
\text { SMU.1815 }\end{array}$ & Bacteriocin production* & {$[49]^{*}$} \\
\hline TCS-6 & $\begin{array}{l}\text { HK- } \\
\text { SpaK } \\
\text { RR- } \\
\text { SpaR }\end{array}$ & $\begin{array}{l}\text { SMU.660 } \\
\text { SMU.659 }\end{array}$ & Bacteriocin production*, self-protection against anti-microbial peptides*. & {$[50-52]^{*}$} \\
\hline TCS-7 & $\begin{array}{l}\text { HK- } \\
\text { PhoR } \\
\text { RR-YcbL }\end{array}$ & $\begin{array}{l}\text { SMU.1037 } \\
\text { SMU.1038 }\end{array}$ & Unknown. & \\
\hline TCS-8 & $\begin{array}{l}\text { HK-KinG } \\
\text { RR-LIrG }\end{array}$ & $\begin{array}{l}\text { SMU.1009 } \\
\text { SMU.1008 }\end{array}$ & Bacteriocin resistance, substrate transport in cell envelope stress. & {$[53,54]$} \\
\hline TCS-9 & $\begin{array}{l}\text { HK-LevS } \\
\text { RR-LevR }\end{array}$ & $\begin{array}{l}\text { SMU.1965 } \\
\text { SMU.1964 }\end{array}$ & Biofilm formation, acid tolerance, fructan metabolism. & {$[55,56]$} \\
\hline TCS-10 & $\begin{array}{l}\text { HK-LytS } \\
\text { RR-LytT }\end{array}$ & $\begin{array}{l}\text { SMU.577 } \\
\text { SMU.576 }\end{array}$ & Biofilm formation, oxidative stress tolerance, autolysis, fructan metabolism, cell wall metabolism*. & {$[57]^{*},[48,58]$} \\
\hline TCS-11 & $\begin{array}{l}\text { HK-LiaS } \\
\text { RR-LiaR }\end{array}$ & $\begin{array}{l}\text { SMU.486 } \\
\text { SMU.487 }\end{array}$ & $\begin{array}{l}\text { Biofilm formation, acid tolerance, cell envelope stress response, bacteriocin production, } \\
\text { bacteriocin resistance, sucrose-dependent adherence. }\end{array}$ & {$[37,59-62]$} \\
\hline TCS-12 & $\begin{array}{l}\text { HK-HK11 } \\
\text { RR-RR11 }\end{array}$ & $\begin{array}{l}\text { SMU.1548 } \\
\text { SMU.1547 }\end{array}$ & Unknown. & \\
\hline TCS-13 & $\begin{array}{l}\text { HK- } \\
\text { ComD } \\
\text { RR- } \\
\text { ComE }\end{array}$ & $\begin{array}{l}\text { SMU.1916 } \\
\text { SMU.1917 }\end{array}$ & Biofilm formation, quorum sensing, competence development, bacteriocin production. & {$[9,44,63-65]$} \\
\hline TCS-14 & $\begin{array}{l}\text { HK } \\
\text { RR }\end{array}$ & $\begin{array}{l}\text { SMU.45 } \\
\text { SMU.46 }\end{array}$ & Unknown. & \\
\hline $\begin{array}{l}\text { Orhpan } \\
\text { RR1 }\end{array}$ & RR-GerR & SMU.1924 & Sucrose-dependent adherence, glucan metabolism, pH homeostasis, competence development. & {$[66-72]$} \\
\hline
\end{tabular}

In case no knowledge is available on the functions of some TCSs in UA159, reports on homologous TCSs in other microorganisms, preferably Streptococcus species (highlighted by the astral sign*) are provided.

in the 10 strains studied, we found that $S$. mutans KK23 and $S$. mutans NN2025 carried two and three genes, respectively, which encode putative bacteriocin precursor peptides sharing more than $60 \%$ similarity with SAFF22. In addition, a putative bacteriocin biosynthesis protein coding gene was also found downstream of the scnKR operon in the two S. mutans strains. We therefore infer that TCS-5 (ScnKR) might be involved in the regulation of mutacin production at least in S. mutans KK23 and S. mutans NN2025.

TCS-7 (PhoR/YcbL) was only shared by the eight $S$. mutans strains. In S. mutans UA159, an insertion mutant of the gene encoding PhoR displayed no significant difference to the wild type with respect to growth under various stress conditions [6]. The clear function of TCS-7 is still unknown.

As mentioned before in the identification and classification of TCS proteins, TCS-13 (ComDE) was absent in S. ratti DSM20564. This finding was also supported by the PCR experiment (Figure 4). In S. mutans, ComDE is the most extensively studied two-component signaling system involved in quorum sensing and competence development. Mediated by the so-called competence stimulating peptide (CSP), it is involved in multiple stress responses and has been implicated in competence development, bacteriocin production, virulence, biofilm formation, and cariogenicity [9,44,63-65]. Further analysis showed that the ortholog of the $\operatorname{com} C$ gene, whose 


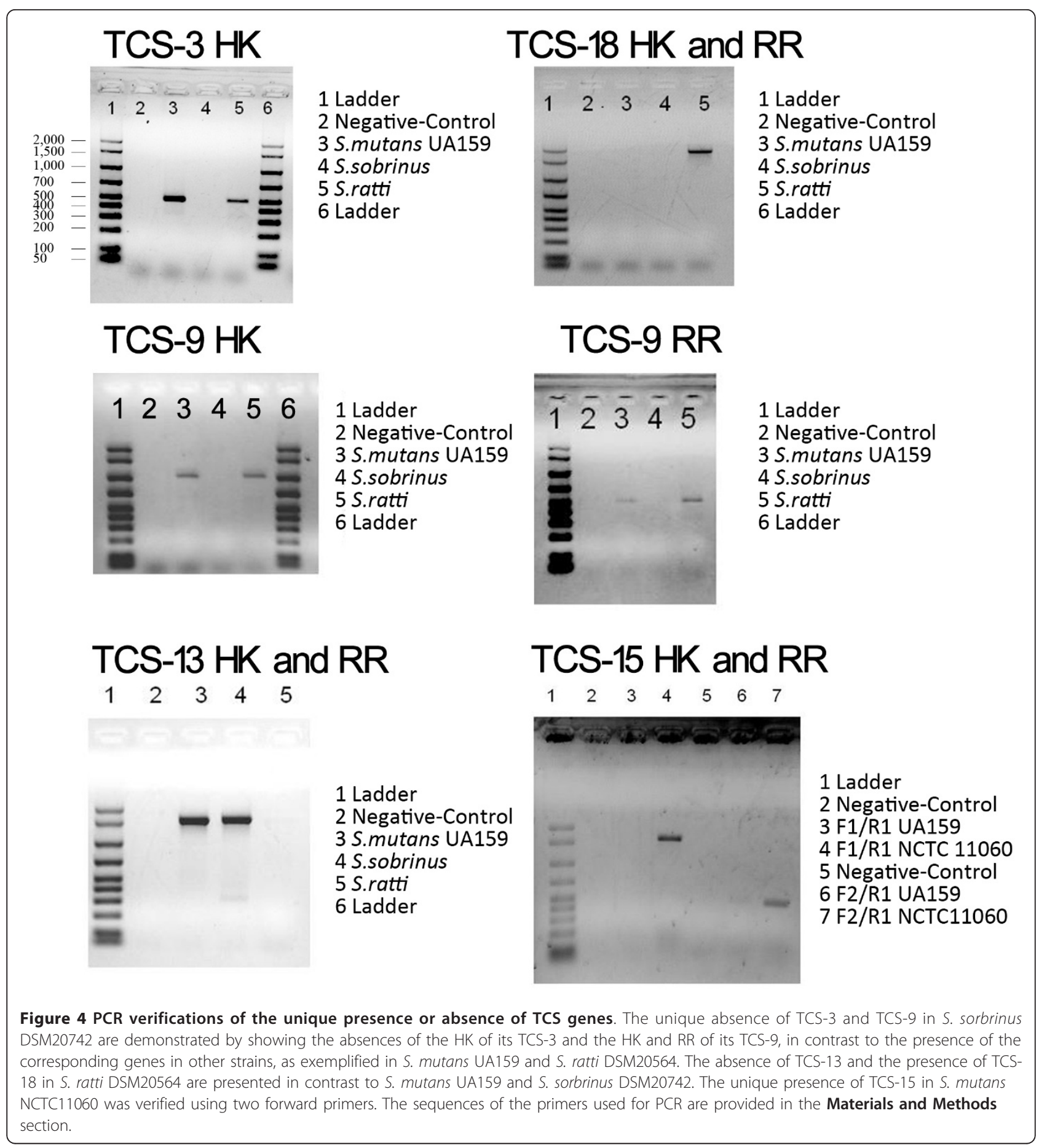

product is the precursor of the signal peptide CSP sensed by ComDE in S. mutans, was absent in S. ratti. Putative orthologs of $c o m D$ and comE were found in $S$. sobrinus DSM20742. However, with a similarity of merely $37 \%$ and $43 \%$, respectively, to the comD and comE of S. mutans UA159 (Table 3), it's highly possible that the actual function of TCS-13 in S. sobrinus DSM20742 might be quite different from that known for $S$. mutans. It is also worthy to mention that no comC-like gene was found in S. sobrinus DSM20742.

Putative alleles of the HK and RR proteins of TCS-14 are present in five of the $S$. mutans strains, namely 5 DC8, KK23, KK21, UA159 and NN2025 (Table 3). This cluster was first identified in S. mutans UA159 by Biswas et al. and the corresponding $H K$ and RR are encoded by SMU.45 and SMU.46, respectively [6]. They 
also found that TCS-14 was present only in two of the $13 \mathrm{~S}$. mutans strains compared in their study. HKs of this TCS cluster contain only one recognizable HATPase_c domain (Figure 3). In addition, no known output domain was identified in the cognate RRs. Thus, neither HKs nor RRs of TCS-14 could be classified into any known HK and RR families. In addition, as depicted by the multi-sequence alignment of the putative TCS-14 HK alleles in Additional file 1, the open reading frame predictions carried out in our study revealed that while SMU.45 and its upstream gene SMU.44 clearly constitute two separate genes in S. mutans UA159, they are merged to constitute the parts of a single gene coding for the HKs in the S. mutans strains 5 DC8, KK23 and NN2025. The corresponding HK of KK21 was also split into two proteins by the lacking of a single glutamine (Q) residue.

TCS-15 was found exclusively in the genome of the serotype f blood isolate S. mutans NCTC11060 (Table 3 ). Genes of a TCS located on the genomic island TnSmu2 of S. mutans UA140, as recently reported by the research group Qi [73], could be possible alleles of TCS-15 genes. This was based on the fact that the predicted HKs and RRs of both TCS 15 in our study and the TCS found by Qi's group have the HK (YP_002747386.1) and RR (YP_002124238.1) of Streptococcus equi, respectively, as the best matched homologs. The HK (smh|00177) of TCS-15 is also the only histidine kinase found in this study that contains a PDZ domain (Figure 3). PDZ domain was first reported to be present in animals. In 1997, Ponting et al. claimed that PDZ domains exist also in diverse signaling proteins of bacteria, yeasts and plants. Experimental evidence was first provided by Liao et al. through the determination of the first crystal structure of a bacterial PDZ domain [74]. The most remarkable feature of PDZ domains is their ability to specifically recognize and bind to short $\mathrm{C}$-terminal peptide motifs. This allows them especially to bind membrane proteins such as ion channels, which have very small free $\mathrm{C}$-termini. To exclude the possibility of contamination with e. g. human DNA during the process of genome sequencing, the existence of the gene coding for this unusual PDZ domain-containing HK protein in the DNA of the NCTC11060 strain was experimentally verified by using two different forward primers in the PCR experiment (Figure 4).

The PDZ domain of smh|00177 is flanked by one transmembrane helix (TM) at its $\mathrm{N}$-terminal side and 9 TMs at its C-terminal side, which is characteristic of a ComP-like HK. ComP-like HKs are a group of sensors of another peptide-dependent quorum sensing system related to cell density-responsive regulation other than ComDE in gram-positive bacteria. In B. subtilis, ComP is the sensor histidine kinase of the four-component
comQXPA quorum sensing system, where ComA stands for the corresponding response regulator, $\operatorname{ComX}$ is the pheromone precursor and ComQ the protein required for the proteolytic cleavage and modification of the pheromone precursor molecule [75]. The most similar homolog of smh $\mid 00177$ found from a BLASTp search in the NCBI database http://blast.ncbi.nlm.nih.gov/ with a sequence identity of around $65 \%$ is the histidine kinase (YP_003353659.1) of a TCS from Lactococcus lactis subsp. lactis KF147. Furthermore, we found that the cognate response regulator of TCS-15 showed a sequence identity of around $78 \%$ with the corresponding response regulator (YP_003353660.1) from the same $L$. lactis subsp. lactis strain. In our study, the RR ( $\mathrm{smh}$ | 00177) of TCS-15 was termed as CmpR (Table 3). It should be mentioned that neither L. lactis subsp. lactis KF147 nor S. mutans NCTC11060 possesses homologs of the B. subtilis $\operatorname{com} X$ and $\operatorname{com} Q$. Thus, the signal peptide sensed by ComP/CmpR in S. mutans NCTC11060 remains unknown.

TCS-16, 17 and 18 are uniquely present in S. ratti DSM20564 (Table 3). According to BLASTp searches against the NCBI database, the top matches to the TCS16 HK protein (sra|800020) or the RR protein (sra| 800019) are all from Streptococcus species such as Streptococcus infantarius and Streptococcus agalactiae. In addition, the HK and RR homologs in the different Streptococcus species are all encoded by two adjacent genes and annotated in some $S$. agalactiae strains as sensor histidine kinase DltS and DNA-binding response regulator DltR. The DltSR has been reported to be involved in the regulation of D-alanyl-lipoteichoic acid biosynthesis in S. agalactiae [76]. Lipoteichoic acid (LTA) is a major cell wall constituent of Gram-positive bacteria which is phosphoglycerol substituted with a DAla ester or a glycosyl residue and anchored in the membrane by its glycolipid moiety. D-alanylation of lipoteichoic acid has been proven to contribute to the virulence of Streptococcus suis [77], as well as to the biofilm formation and resistance to antimicrobial peptides in enterococci [78]. Thus, the TCS-16 cluster might also be an important virulence factor in S. ratti.

The TCS-17 was composed of a HAMP-containing HK and an OmpR-type RR. The best homologs of the HK protein (sra|3500015) and the RR protein (sra| 3500014) are from S. agalactiae strains. But the functions of these homologous proteins remain unknown.

TCS-18 is the only cluster that comprises a HPK 11 family $H K$ and an AmiR family RR, which possesses a novel RNA-binding type output domain. The top ten best hits of the BLASTp search in the NCBI database showed that the closest homologs for the HK and RR of TCS-18 are all from Listeria species. Since the HK of TCS-18 possesses a PAS domain that is commonly 
involved in sensing intracellular signals such as redox potential, similar to the sensing mechanism described for the HK (VicK) of TCS-1, the function of TCS-18 might also be related to the sensing and response to signal(s) originated in the cytoplasm. The unique presence of TCS-18 in S. ratti DSM20564 was also confirmed by the PCR experiment (Figure 4).

\section{Conclusions}

In the present study we conducted a genome-wide identification, classification, and ortholog analysis of the TCS proteins in eight newly sequenced mutans streptococci strains in comparison with two previously sequenced $S$. mutans strains, UA159 and NN2025. Totally, 18 TCS clusters comprising HK-RR pairs were identified, with 8 of them shared by all the 10 strains compared, 6 being absent in one or more strains, 1 unique to $S$. mutans NCTC11060 and 3 exclusive to $S$. ratti DSM20564. S. mutans strains share to a large extend the same TCS repertoires. One remarkable exception is the unique presence of the putative quorum-sensing TCS-15 (ComP/CmpR) in the only serotype f blood isolate, S. mutans NCTC11060. S. sobrinus DSM20742 shows differences to the $S$. mutans strains in the signal perceptions possibly related to fructan catabolism and acid tolerance response. With its 3 unique TCS clusters, $S$. ratti DSM20564 seems even more distinct to the $S$. mutans strains. One of the unique TCS clusters is homologous to the DltSR TCS in Streptococcus agalactiae strains that is probably involved in the regulation of the biosynthesis of $\mathrm{D}$-alanyl-lipoteichoic acid, an important cell wall component. Another one has an AmiR-like $R R$ protein, which is the only $R R$ found in this study to contain a RNA-binding output domain instead of a DNAbinding output domain.

In addition to highlighting putative functions of the individual histidine kinases and response regulators, our detailed classification of the TCS proteins also confirms that specific HK families are preferentially associated with specific RR families. Such associations might play significant roles in the phenomenon of cross-talking between unpaired HKs and RRs.

The asymmetric distribution of TCS among the mutans streptococci strains compared in this study implies that $S$. mutans, S. sobrinus and S. ratti might employ different sensing and response mechanisms for their survival in the fast changing oral environment, mostly in a symbiotic lifestyle. We believe that the results from this genomic level study will be certainly helpful for the design of physiological studies and mutation analysis towards the verification of some hypotheses proposed in this study, which in turn will lead to a better understanding of signal transductions involved in the pathogenicities of mutans streptococci.

\section{Methods}

\section{Mutans streptococci strains sequenced}

We have recently sequenced eight mutans streptococci strains as given in Table 1 using the Solexa sequencing platform at the Helmholtz Center for Infection Research in Braunschweig, Germany. The "high-quality draft" genome sequences of these mutans streptococci strains were assembled by a combined use of the sequence assembly tools SOAPdenovo [79], Maq [80] and Phrap [81]. Corresponding protein sequences were predicted using Glimmer3.02, a gene finding tool based on interpolated Markov models [82]. Detailed genome sequence information is available at http://134.28.64.65/www/ index.php/genome.

\section{Identification of the histidine kinases and response regulators of putative two-component signal transduction systems}

The identification of histidine kinases (HKs) and response regulators (RRs) of putative two-component systems (TCSs) of the eight mutans streptococci strains was carried out based on computational domain analysis of the predicted protein sequences. Two previously sequenced $S$. mutans strains, the $S$. mutans UA159 and S. mutans NN2025, were used as reference strains for comparison. To this end, the same identification procedure was carried out on the genomes of S. mutans NN2025 and UA159 to ensure that the same search criteria were applied for all the strains included in this study so that a reasonable comparison can be achieved. The genome sequences of the two reference strains were obtained from the genome database at the National Center for Biotechnology Information http:// www.ncbi.nlm.nih.gov/sites/genome. Approaches for identifying $\mathrm{HKs}$ and RRs were similar to those described previously [83] with slight modifications. Briefly, putative HK and RR proteins were identified by Hidden Markov Model (HMM) searches using the related HMM profiles available in the Pfam database http:// pfam.sanger.ac.uk/ as templates [14]. The sequence homology search software HMMER3 http://hmmer.org/ [84] was used for scanning the predicted protein sequences with the HMM profiles. All the HK related HMM profiles with the accession numbers PF00512, PF07568, PF07730, PF07536, PF06580, PF01627, PF02895, PF05384, PF10090 were used for identifying putative HKs. The HMM profile PF00072 which targets the receiver (REC) domain of RR proteins was used to recognize putative RRs. For the identification of HKs, the homology search was performed without setting Evalue/score cutoffs to avoid missing any putative HKs with low scores. However, all the identified putative HKs were manually validated by judging whether at 
least one of the following two criteria was satisfied: (a) the presence of a cognate putative $R R$ in the same operon as the putative HK in question; (b) the presence of both the HisKA-like and HATPase_c domains so that any HATPase_c domain possessing non-HK proteins could be excluded. For the identification of putative RRs, the E-value cut-off was set at 1e-6. Paired HK and RR present in the same operon comprise a TCS cluster. Hybrid HKs, if any, could be determined by the presence of a complete HK transmitter domain and a REC domain in a single protein. HKs and RRs are defined as orphan HKs or RRs, if no corresponding cognate RRs or HKs can be found in the same operon. The operon information used in this study was predicted by Pathway Tools [85].

\section{Identification of common and unique TCS proteins}

TCS proteins that are common or unique among the mutans streptococci strains were identified through ortholog analysis. The ortholog groups were constructed by using the OrthoMCL program [86]. OrthoMCL was first developed by focusing on eukaryotic genomes and now has been successfully applied to prokaryotic genomes as well. BLASTp [87] was employed to construct the homologous protein matrices which were used in the ortholog group identification process by OrthoMCL with an E-value cutoff of 1e-5.

\section{Classification of the TCS proteins}

Functional domains of the TCS proteins, which were needed for the classification of both HKs and RRs were obtained by subjecting the predicted proteome of each strain to a search against the protein sequence analysis and classification database InterPro using the protein signature recognition software InterProScan [88]. In addition, for the classification of HK proteins, transmembrane helices were predicted by using the hidden Markov model based program TMHMM2 [89].

The HK protein classifications were carried out by two different approaches: a) homology box based analysis through the recognition and alignment of characteristic sequence motifs (homology boxes) of different HK families, as described previously by Grebe et al. [15]; b) domain architecture based analysis of the transmembrane regions and domain organization by a method described previously by Mascher et al. [13]. As the SMART database http://smart.embl-heidelberg.de provided a user-friendly web interface and excellent illustration features, the visualization of HK transmembrane helices, conserved domains and their organization was realized by using the SMART web interface with small manual modifications for the pictorial depictions.

\section{Co-evolution analysis of paired HKs and RRs}

All the putative HKs and RRs identified in the mutans streptococci strains compared in this study were used for establishing the phylogenetic relationships between the TCS proteins. Using the ClustalX program multiple alignments were performed with the following parameter settings: residue-specific penalties: on, hydrophilic penalties: on, gap separation distance: 4 and the phylogenetic relationships with a bootstrap value set at 1000 were constructed [90]. The results were imported into the visualization software Treeview [91] for the visualization of the phylogenetic trees of HKs and RRs, respectively.

\section{PCR verification}

To verify the unique presence of TCS-15 in S. mutans NCTC11060 and to exclude the possibility of contamination with e. g. human DNA during the process of genome sequencing, PCR amplification with original DNA from this strain (and from strain S. mutans UA159 as negative control) using two different forward primers was performed. The primers used were: $5^{\prime}$ TTGCTTGCTGTTGTTGTG-3' (forward primer), 5'GGCTACCATTTAGTAGAAAAGAGG -3' (alternative forward primer), 5'-TGTTACCATCTTCGGAAGG-3' (reverse primer), which were designed by using Primer Premier 6 http://www.premierbiosoft.com/primerdesign/ index.html and Vector NTI 9.0 (InforMax) respectively. Conditions for this conventional PCR were: $94^{\circ} \mathrm{C}, 2 \mathrm{~min}$; followed by 32 cycles of $94^{\circ} \mathrm{C}$ for $30 \mathrm{~s}$; annealing temperature $49^{\circ} \mathrm{C}$ for $30 \mathrm{~s}$; and $72^{\circ} \mathrm{C}$ for $90 \mathrm{~s}$; final extension at $72^{\circ} \mathrm{C}$ for $5 \mathrm{~min}$; length of amplicons: $1624 \mathrm{bp}$ and 504 $\mathrm{bp}$, respectively.

To verify the unique presence of TCS-18 in S. ratti, the unique absence of TCS-13 in S. ratti, and the unique absence of TCS-9 as well as TCS-3 in S. sobrinus, PCR amplification using original DNA from strains S. mutans UA159, S. ratti DSM20564, and S. sobrinus DSM20742 was performed. The primers used, the annealing temperatures and the lengths of amplicons were as follows (all other parameters were kept as mentioned above): TCS-18 F 5' CACTGTTCCTCCTGTATCC 3', TCS-18R 5' ATGCTGGCTATGATGTTGT 3' $\left(\mathrm{T}_{\mathrm{a}}=50^{\circ} \mathrm{C}\right.$, length: 1899 bp covering $\mathrm{HK}$ and $\left.\mathrm{RR}\right)$; TCS-13 F 5' RAKTTYATGCCYCTMACYTTYCAG 3', TCS-13R 5' GATTCRWWRGCMGCCTC 3' $\left(\mathrm{T}_{\mathrm{a}}=49^{\circ} \mathrm{C}\right.$, length: 1600 bp covering HK and RR); TCS-9 HK-F 5' ATACAGTCAATATGCYAAGC 3', TCS-9HK-R 5' GRATAACACGGAAAA 3' $\left(\mathrm{T}_{\mathrm{a}}=45 \mathrm{C}\right.$, length: 1055 bp); TCS-9 RR-F 5' TGCTGARGACCAAGA 3', TCS9RR-R 5' TTAGCTGCAATTTCTT 3' $\left(\mathrm{T}_{\mathrm{a}}=50^{\circ} \mathrm{C}\right.$, length: 522 bp); TCS-3 HK-F 5' CAYGAYYTIMGIAAYCC 3', TCS-3 HK-R 5' GTDATIACIGTICCC 3' $\left(\mathrm{T}_{\mathrm{a}}=40^{\circ} \mathrm{C}\right.$, length: $\left.505 \mathrm{bp}\right)$. 


\section{Additional material}

Additional file 1: Multiple sequence alignment of the allelic HK proteins belonging to the TCS-14 cluster. smd|00044 and smd|00045 from S. mutans KK21; sme|00049 from S. mutans KK23; smc|00046 from S. mutans 5DC8; SMU.44 and SMU.45 from S. mutans UA159; Gl|290579565 from S. mutans NN2025. The splitting of the alleles into two separate proteins in the strains S. mutans KK21 and S. mutans UA159 can be observed.

\section{Abbreviations}

TCS: Two component system; HK: Sensing histidine kinase; RR: Response regulator; HTH: Helix-turn-helix; HMM: Hidden Markov Model; HPT: Histidine phosphotransferases; TM: Transmembrane helix; HGT: Horizontal gene transfer; PCR: Polymerase Chain Reaction.

\section{Acknowledgements}

This study was done within the project "Development of biofilm inhibitors using a systems biology approach" (0315411) which is financed by the German Federal Ministry of Education and Research (BMBF) in the frame of the Research Program "Medical systems biology - MedSys".

\section{Author details}

${ }^{1}$ Institute of Bioprocess and Biosystems Engineering, Hamburg University of Technology, Hamburg, Germany. ${ }^{2}$ Division of Oral Microbiology and Immunology, Department of Operative and Preventive Dentistry \& Periodontology, RWTH Aachen University, Aachen, Germany. ${ }^{3}$ Group Microbial Communication, Division of Microbial Pathogenesis, HelmholtzCentre for Infection Research, Inhoffenstr 7, 38124, Braunschweig, Germany. ${ }^{4}$ Tianjin Institute of Industrial Biotechnology, Chinese Academy of Science, Tianjin 300308, Peoples Republic of China.

\section{Authors' contributions}

LS carried out the major bioinformatics analysis and participated in drafting the manuscript. PS was involved in the bioinformatic analysis and contributed significantly to drafting the manuscript. WW participated in the conception and coordination of the study and contributed significantly to drafting the manuscript. $G C$ and $A B$ performed the $P C R$ verification experiments. JS was involved in genome sequencing and annotation. GC and IWD contributed to the microbial aspects and valuable discussions. AZE conceived of and supervised the study and revised the manuscript. All authors read and approved the final manuscript.

\section{Competing interests}

The authors declare that they have no competing interests.

Received: 10 August 2011 Accepted: 4 April 2012

Published: 4 April 2012

\section{References}

1. Lemos JA, Burne RA: A model of efficiency: stress tolerance by Streptococcus mutans. Microbiology 2008, 154(Pt 11):3247-3255.

2. Nakano K, Nomura R, Matsumoto M, Ooshima T: Roles of oral bacteria in cardiovascular diseases-from molecular mechanisms to clinical cases: Cell-surface structures of novel serotype k Streptococcus mutans strains and their correlation to virulence. J Pharmacol Sci 2010, 113(2):120-125.

3. Stock AM, Robinson VL, Goudreau PN: Two-component signal transduction. Annu Rev Biochem 2000, 69:183-215.

4. Fabret C, Feher VA, Hoch JA: Two-component signal transduction in Bacillus subtilis: how one organism sees its world. J Bacteriol 1999, 181(7):1975-1983.

5. Ajdic D, McShan WM, McLaughlin RE, Savic G, Chang J, Carson MB, Primeaux C, Tian R, Kenton S, Jia H, et al: Genome sequence of Streptococcus mutans UA159, a cariogenic dental pathogen. Proc Natl Acad Sci USA 2002, 99(22):14434-14439.

6. Biswas I, Drake L, Erkina D, Biswas S: Involvement of sensor kinases in the stress tolerance response of Streptococcus mutans. J Bacteriol 2008, 190(1):68-77.
7. Liu Y, Burne RA: Multiple two-component systems of Streptococcus mutans regulate agmatine deiminase gene expression and stress tolerance. J Bacteriol 2009, 191(23):7363-7366.

8. Lee SF, Delaney GD, Elkhateeb M: A two-component covRS regulatory system regulates expression of fructosyltransferase and a novel extracellular carbohydrate in Streptococcus mutans. Infect Immun 2004, 72(7):3968-3973.

9. van der Ploeg JR: Regulation of bacteriocin production in Streptococcus mutans by the quorum-sensing system required for development of genetic competence. J Bacterio/ 2005, 187(12):3980-3989.

10. Senadheera MD, Guggenheim B, Spatafora GA, Huang YC, Choi J, Hung DC, Treglown JS, Goodman SD, Ellen RP, Cvitkovitch DG: A VicRK signal transduction system in Streptococcus mutans affects gtfBCD, gbpB, and $\mathrm{ftf}$ expression, biofilm formation, and genetic competence development. J Bacteriol 2005, 187(12):4064-4076.

11. Chain PS, Grafham DV, Fulton RS, Fitzgerald MG, Hostetler J, Muzny D, Ali J, Birren B, Bruce DC, Buhay C, et al: Genomics. Genome project standards in a new era of sequencing. Science 2009, 326(5950):236-237.

12. Levesque CM, Mair RW, Perry JA, Lau PC, Li YH, Cvitkovitch DG: Systemic inactivation and phenotypic characterization of two-component systems in expression of Streptococcus mutans virulence properties. Lett Appl Microbiol 2007, 45(4):398-404.

13. Mascher T, Helmann JD, Unden G: Stimulus perception in bacterial signaltransducing histidine kinases. Microbiol Mol Biol Rev 2006, 70(4):910-938.

14. Finn RD, Mistry J, Tate J, Coggill P, Heger A, Pollington JE, Gavin OL, Gunasekaran P, Ceric G, Forslund K, et al: The Pfam protein families database. Nucleic Acids Res 2010, 38(Database):211-222.

15. Grebe TW, Stock JB: The histidine protein kinase superfamily. Adv Microb Physiol 1999, 41:139-227.

16. Aravind L, Ponting CP: The cytoplasmic helical linker domain of receptor histidine kinase and methyl-accepting proteins is common to many prokaryotic signalling proteins. FEMS Microbiol Lett 1999, 176(1):111-116.

17. Taylor BL, Zhulin IB: PAS domains: internal sensors of oxygen, redox potential, and light. Microbiol Mol Biol Rev 1999, 63(2):479-506.

18. Nguyen AN, Lee A, Place W, Shiozaki K: Multistep phosphorelay proteins transmit oxidative stress signals to the fission yeast stress-activated protein kinase. Mol Biol Cell 2000, 11(4):1169-1181.

19. Echenique $J R$, Trombe MC: Competence repression under oxygen limitation through the two-component MicAB signal-transducing system in Streptococcus pneumoniae and involvement of the PAS domain of MicB. J Bacteriol 2001, 183(15):4599-4608.

20. Rickman L, Saldanha JW, Hunt DM, Hoar DN, Colston MJ, Millar JB, Buxton RS: A two-component signal transduction system with a PAS domain-containing sensor is required for virulence of Mycobacterium tuberculosis in mice. Biochem Biophys Res Commun 2004, 314(1):259-267.

21. Shin JH, Lee HW, Kim SM, Kim J: Proteomic analysis of Acinetobacter baumannii in biofilm and planktonic growth mode. J Microbiol 2009, 47(6):728-735.

22. Zhulin IB, Taylor BL, Dixon R: PAS domain S-boxes in Archaea, Bacteria and sensors for oxygen and redox. Trends Biochem Sci 1997, 22(9):331-333.

23. Hefti MH, Francoijs KJ, de Vries SC, Dixon R, Vervoort J: The PAS fold. A redefinition of the PAS domain based upon structural prediction. Eur $J$ Biochem 2004, 271(6):1198-1208.

24. Deng DM, Liu MJ, ten Cate JM, Crielaard W: The VicRK system of Streptococcus mutans responds to oxidative stress. J Dent Res 2007, 86(7):606-610.

25. Aravind L, Ponting CP: The GAF domain: an evolutionary link between diverse phototransducing proteins. Trends Biochem Sci 1997 22(12):458-459.

26. Zoraghi R, Corbin JD, Francis SH: Properties and functions of GAF domains in cyclic nucleotide phosphodiesterases and other proteins. Mol Pharmacol 2004, 65(2):267-278.

27. Mascher T: Intramembrane-sensing histidine kinases: a new family of cell envelope stress sensors in Firmicutes bacteria. FEMS Microbiol Lett 2006, 264(2):133-144.

28. Mascher T, Margulis NG, Wang T, Ye RW, Helmann JD: Cell wall stress responses in Bacillus subtilis: the regulatory network of the bacitracin stimulon. Mol Microbiol 2003, 50(5):1591-1604.

29. Pao GM, Saier MH Jr: Response regulators of bacterial signal transduction systems: selective domain shuffling during evolution. J Mol Evol 1995, 40(2):136-154. 
30. Galperin MY: Structural classification of bacterial response regulators: diversity of output domains and domain combinations. J Bacteriol 2006, 188(12):4169-4182.

31. O'Hara BP, Norman RA, Wan PT, Roe SM, Barrett TE, Drew RE, Pearl LH: Crystal structure and induction mechanism of AmiC-AmiR: a ligandregulated transcription antitermination complex. EMBO J 1999, 18(19):5175-5186.

32. Martinez-Hackert E, Stock AM: The DNA-binding domain of OmpR: crystal structures of a winged helix transcription factor. Structure 1997, 5(1):109-124.

33. Rodriguez-Moya J, Argandona M, Reina-Bueno M, Nieto JJ, Iglesias-Guerra F, Jebbar M, Vargas C: Involvement of EupR, a response regulator of the $\mathrm{NarL/FixJ} \mathrm{family,} \mathrm{in} \mathrm{the} \mathrm{control} \mathrm{of} \mathrm{the} \mathrm{uptake} \mathrm{of} \mathrm{the} \mathrm{compatible} \mathrm{solutes}$ ectoines by the halophilic bacterium Chromohalobacter salexigens. BMC Microbiol 2010, 10:256.

34. Nikolskaya AN, Galperin MY: A novel type of conserved DNA-binding domain in the transcriptional regulators of the AlgR/AgrA/LytR family. Nucleic Acids Res 2002, 30(11):2453-2459.

35. Galperin MY: Telling bacteria: do not LytTR. Structure 2008, 16(5):657-659.

36. Mitrophanov AY, Groisman EA: Signal integration in bacterial twocomponent regulatory systems. Genes Dev 2008, 22(19):2601-2611.

37. Li YH, Lau PC, Tang N, Svensater G, Ellen RP, Cvitkovitch DG: Novel twocomponent regulatory system involved in biofilm formation and acid resistance in Streptococcus mutans. J Bacteriol 2002, 184(22):6333-6342.

38. Bhagwat SP, Nary J, Burne RA: Effects of mutating putative twocomponent systems on biofilm formation by Streptococcus mutans UA159. FEMS Microbiol Lett 2001, 205(2):225-230.

39. Wagner C, Saizieu Ad A, Schonfeld HJ, Kamber M, Lange R, Thompson CJ, Page MG: Genetic analysis and functional characterization of the Streptococcus pneumoniae vic operon. Infect Immun 2002, 70(11):6121-6128.

40. Tremblay YD, Lo H, Li YH, Halperin SA, Lee SF: Expression of the Streptococcus mutans essential two-component regulatory system VicRK is $\mathrm{pH}$ and growth-phase dependent and controlled by the LiaFSR threecomponent regulatory system. Microbiology 2009, 155(Pt 9):2856-2865.

41. Senadheera D, Krastel K, Mair R, Persadmehr A, Abranches J, Burne RA Cvitkovitch DG: Inactivation of VicK affects acid production and acid survival of Streptococcus mutans. J Bacterio/ 2009, 191(20):6415-6424.

42. Ahn SJ, Burne RA: Effects of oxygen on biofilm formation and the AtIA autolysin of Streptococcus mutans. J Bacteriol 2007, 189(17):6293-6302.

43. Ahn SJ, Wen ZT, Burne RA: Effects of oxygen on virulence traits of Streptococcus mutans. J Bacteriol 2007, 189(23):8519-8527.

44. Ahn SJ, Wen ZT, Burne RA: Multilevel control of competence development and stress tolerance in Streptococcus mutans UA159. Infect Immun 2006, 74(3):1631-1642.

45. Qi F, Merritt J, Lux R, Shi W: Inactivation of the ciaH Gene in Streptococcus mutans diminishes mutacin production and competence development, alters sucrose-dependent biofilm formation, and reduces stress tolerance. Infect Immun 2004, 72(8):4895-4899.

46. Chen PM, Chen HC, Ho CT, Jung CJ, Lien HT, Chen JY, Chia JS: The twocomponent system ScnRK of Streptococcus mutans affects hydrogen peroxide resistance and murine macrophage killing. Microbes Infect 2008, 10(3):293-301

47. Lemos JA, Lin VK, Nascimento MM, Abranches J, Burne RA: Three gene products govern (p)ppGpp production by Streptococcus mutans. Mol Microbiol 2007, 65(6):1568-1581.

48. Kawada-Matsuo M, Shibata Y, Yamashita Y: Role of two component signaling response regulators in acid tolerance of Streptococcus mutans. Oral Microbiol Immunol 2009, 24(2):173-176.

49. MCLaughlin RE, Ferretti JJ, Hynes WL: Nucleotide sequence of the streptococcin A-FF22 lantibiotic regulon: model for production of the lantibiotic SA-FF22 by strains of Streptococcus pyogenes. FEMS Microbiol Lett 1999, 175(2):171-177.

50. Kleerebezem M, Quadri LE, Kuipers OP, de Vos WM: Quorum sensing by peptide pheromones and two-component signal-transduction systems in Gram-positive bacteria. Mol Microbiol 1997, 24(5):895-904.

51. Klein C, Entian KD: Genes involved in self-protection against the lantibiotic subtilin produced by Bacillus subtilis ATCC 6633. App/ Environ Microbiol 1994, 60(8):2793-2801
52. Klein C, Kaletta C, Entian KD: Biosynthesis of the lantibiotic subtilin is regulated by a histidine kinase/response regulator system. Appl Environ Microbiol 1993, 59(1):296-303

53. Tsuda H, Yamashita $Y$, Shibata $Y$, Nakano $Y$, Koga $T$ : Genes involved in bacitracin resistance in Streptococcus mutans. Antimicrob Agents Chemother 2002, 46(12):3756-3764.

54. Ouyang J, Tian XL, Versey J, Wishart A, Li YH: The BceABRS fourcomponent system regulates the bacitracin-induced cell envelope stress response in Streptococcus mutans. Antimicrob Agents Chemother 2010, 54(9):3895-3906.

55. Zeng L, Wen ZT, Burne RA: A novel signal transduction system and feedback loop regulate fructan hydrolase gene expression in Streptococcus mutans. Mol Microbiol 2006, 62(1):187-200.

56. Zeng L, Das S, Burne RA: Genetic Analysis of the Functions and Interactions of Components of the LevQRST Signal Transduction Complex of Streptococcus mutans. PLoS One 2011, 6(2):e17335.

57. Brunskill EW, Bayles KW: Identification and molecular characterization of a putative regulatory locus that affects autolysis in Staphylococcus aureus. J Bacteriol 1996, 178(3):611-618.

58. Ahn SJ, Rice KC, Oleas J, Bayles KW, Burne RA: The Streptococcus mutans Cid and Lrg Systems Modulate Virulence Traits in Response to Multiple Environmental Signals. Microbiology 2010.

59. Chong $P$, Drake L, Biswas I: LiaS regulates virulence factor expression in Streptococcus mutans. Infect Immun 2008, 76(7):3093-3099.

60. Suntharalingam P, Senadheera MD, Mair RW, Levesque CM, Cvitkovitch DG: The LiaFSR system regulates the cell envelope stress response in Streptococcus mutans. J Bacteriol 2009, 191(9):2973-2984.

61. Zhang J, Biswas I: A phenotypic microarray analysis of a Streptococcus mutans liaS mutant. Microbiology 2009, 155(Pt 1):61-68.

62. Perry JA, Levesque CM, Suntharaligam P, Mair RW, Bu M, Cline RT, Peterson SN, Cvitkovitch DG: Involvement of Streptococcus mutans regulator RR11 in oxidative stress response during biofilm growth and in the development of genetic competence. Lett Appl Microbiol 2008, 47(5):439-444

63. Kreth J, Hung DC, Merritt J, Perry J, Zhu L, Goodman SD, Cvitkovitch DG, Shi W, Qi F: The response regulator ComE in Streptococcus mutans functions both as a transcription activator of mutacin production and repressor of CSP biosynthesis. Microbiology 2007, 153(Pt 6):1799-1807.

64. Li YH, Tang N, Aspiras MB, Lau PC, Lee JH, Ellen RP, Cvitkovitch DG: A quorum-sensing signaling system essential for genetic competence in Streptococcus mutans is involved in biofilm formation. J Bacteriol 2002, 184(10):2699-2708

65. Li YH, Tian XL, Layton G, Norgaard C, Sisson G: Additive attenuation of virulence and cariogenic potential of Streptococcus mutans by simultaneous inactivation of the ComCDE quorum-sensing system and HK/RR11 two-component regulatory system. Microbiology 2008, 154(Pt 11):3256-3265

66. Idone V, Brendtro S, Gillespie R, Kocaj S, Peterson E, Rendi M, Warren W, Michalek S, Krastel K, Cvitkovitch D, et al: Effect of an orphan response regulator on Streptococcus mutans sucrose-dependent adherence and cariogenesis. Infect Immun 2003, 71(8):4351-4360.

67. Biswas I, Drake L, Biswas S: Regulation of gbpC expression in Streptococcus mutans. J Bacteriol 2007, 189(18):6521-6531.

68. Chong P, Chattoraj P, Biswas I: Activation of the SMU.1882 Transcription by CovR in Streptococcus mutans. PLoS One 2010, 5(11):15528.

69. Biswas I, Scott JR: Identification of rocA, a positive regulator of covR expression in the group A streptococcus. J Bacteriol 2003, 185(10):3081-3090.

70. Biswas S, Biswas I: Regulation of the glucosyltransferase ( $\mathrm{gtfBC}$ ) operon by CovR in Streptococcus mutans. J Bacteriol 2006, 188(3):988-998.

71. Chong P, Drake L, Biswas I: Modulation of covR expression in Streptococcus mutans UA159. J Bacteriol 2008, 190(13):4478-4488.

72. Dmitriev A, Mohapatra SS, Chong P, Neely M, Biswas S, Biswas I: CovRControlled Global Regulation of Gene Expression in Streptococcus mutans. PLoS One 2011, 6(5):e20127.

73. Wu C, Cichewicz R, Li Y, Liu J, Roe B, Ferretti J, Merritt J, Qi F: Genomic island TnSmu2 of Streptococcus mutans harbors a nonribosomal peptide synthetase-polyketide synthase gene cluster responsible for the biosynthesis of pigments involved in oxygen and $\mathrm{H} 2 \mathrm{O} 2$ tolerance. Appl Environ Microbiol 2010, 76(17):5815-5826. 
74. Liao DI, Qian J, Chisholm DA, Jordan DB, Diner BA: Crystal structures of the photosystem II D1 C-terminal processing protease. Nat Struct Biol 2000, 7(9):749-753.

75. Ansaldi M, Dubnau D: Diversifying selection at the Bacillus quorumsensing locus and determinants of modification specificity during synthesis of the ComX pheromone. J Bacteriol 2004, 186(1):15-21.

76. Poyart C, Lamy MC, Boumaila C, Fiedler F, Trieu-Cuot P: Regulation of Dalanyl-lipoteichoic acid biosynthesis in Streptococcus agalactiae involves a novel two-component regulatory system. J Bacteriol 2001, 183(21):6324-6334

77. Fittipaldi N, Sekizaki T, Takamatsu D, Harel J: Dominguez-Punaro Mde L, Von Aulock S, Draing C, Marois C, Kobisch M, Gottschalk M: Dalanylation of lipoteichoic acid contributes to the virulence of Streptococcus suis. Infect Immun 2008, 76(8):3587-3594.

78. Fabretti F, Theilacker C, Baldassarri L, Kaczynski Z, Kropec A, Holst O, Huebner J: Alanine esters of enterococcal lipoteichoic acid play a role in biofilm formation and resistance to antimicrobial peptides. Infect Immun 2006, 74(7):4164-4171.

79. Li R, Yu C, Li Y, Lam TW, Yiu SM, Kristiansen K, Wang J: SOAP2: an improved ultrafast tool for short read alignment. Bioinformatics 2009, 25(15):1966-1967.

80. Li H, Durbin R: Fast and accurate short read alignment with BurrowsWheeler transform. Bioinformatics 2009, 25(14):1754-1760,

81. de la Bastide M, McCombie WR: Assembling genomic DNA sequences with PHRAP. Curr Protoc Bioinformatics 2007, Chapter 11(Unit11 14).

82. Delcher AL, Bratke KA, Powers EC, Salzberg SL: Identifying bacterial genes and endosymbiont DNA with Glimmer. Bioinformatics 2007, 23(6):673-679.

83. Lavin JL, Kiil K, Resano O, Ussery DW, Oguiza JA: Comparative genomic analysis of two-component regulatory proteins in Pseudomonas syringae. BMC Genomics 2007, 8:397.

84. Eddy SR: Profile hidden Markov models. Bioinformatics 1998, 14(9):755-763.

85. Karp PD, Paley SM, Krummenacker M, Latendresse M, Dale JM, Lee TJ, Kaipa P, Gilham F, Spaulding A, Popescu L, et al: Pathway Tools version 13.0: integrated software for pathway/genome informatics and systems biology. Brief Bioinform 2010, 11(1):40-79.

86. Li L, Stoeckert CJ Jr, Roos DS: OrthoMCL: identification of ortholog groups for eukaryotic genomes. Genome Res 2003, 13(9):2178-2189.

87. Altschul SF, Madden TL, Schaffer AA, Zhang J, Zhang Z, Miller W, Lipman DJ: Gapped BLAST and PSI-BLAST: a new generation of protein database search programs. Nucleic Acids Res 1997, 25(17):3389-3402.

88. Hunter S, Apweiler R, Attwood TK, Bairoch A, Bateman A, Binns D, Bork P, Das $U$, Daugherty $L$, Duquenne $L$, et al: InterPro: the integrative protein signature database. Nucleic Acids Res 2009, , 37 Database: 211-215.

89. Krogh A, Larsson B, von Heijne G, Sonnhammer EL: Predicting transmembrane protein topology with a hidden Markov model: application to complete genomes. J Mol Biol 2001, 305(3):567-580.

90. Larkin MA, Blackshields G, Brown NP, Chenna R, McGettigan PA, McWilliam H, Valentin F, Wallace IM, Wilm A, Lopez R, et al: Clustal W and Clustal X version 2.0. Bioinformatics 2007, 23(21):2947-2948.

91. Page RD: Visualizing phylogenetic trees using TreeView. Curr Protoc Bioinformatics 2002, Chapter 6(Unit 6 2).

\section{Submit your next manuscript to BioMed Central and take full advantage of:}

- Convenient online submission

- Thorough peer review

- No space constraints or color figure charges

- Immediate publication on acceptance

- Inclusion in PubMed, CAS, Scopus and Google Scholar

- Research which is freely available for redistribution

Submit your manuscript at www.biomedcentral.com/submit 